\title{
Transverse Magnetoresistance of GaAs/AlGaAs Heterojunctions in the Presence of Parallel Magnetic Fields
}

\author{
J.M. Heisz and E. Zaremba \\ Department of Physics, Queen's University, Kingston, Ont., Canada K' 7 L 3N6
}

(October 1, 2018)

\begin{abstract}
We have calculated the resistivity of a GaAs/AlGaAs heterojunction in the presence of both an in-plane magnetic field and a weak perpendicular component using a semiclassical Boltzmann transport theory. These calculations take into account fully the distortion of the Fermi contour which is induced by the parallel magnetic field. The scattering of electrons is assumed to be due to remote ionized impurities. A positive magnetoresistance is found as a function of the perpendicular component, in good qualitative agreement with experimental observations. The main source of this effect is the strong variation of the electronic scattering rate around the Fermi contour which is associated with the variation in the mean distance of the electronic states from the remote impurities. The magnitude of the positive magnetoresistance is strongly correlated with the residual acceptor impurity density in the GaAs layer. The carrier lifetime anisotropy also leads to an observable anisotropy in the resistivity with respect to the angle between the current and the direction of the in-plane magnetic field.
\end{abstract}

PACS Numbers: 73.20.Dx, 73.40.Kp

Typeset using REVTEX 


\section{INTRODUCTION}

Magnetotransport in a two-dimensional electron gas (2DEG) is an extremely rich and complex subject. In the more usual geometry with a magnetic field perpendicular to the plane of the 2DEG, the magnetoresistance exhibits Shubnikov-de Haas oscillations which are the low-field precursors of the quantum Hall effect. This behaviour is a consequence of the quantization of the in-plane orbital motion into Landau levels. The situation with a magnetic field in the plane of the 2DEG is quite different since the cyclotron motion now competes with the effects of the potential confining the electrons. This leads to an interesting modification of the electronic energy band structure which was recently analyzed in detail for typical GaAs/AlGaAs heterojunction,1 1 月. These calculations account for the observed depopulation of higher subbands $\mathbf{B}^{3}$ which occurs with increasing parallel magnetic field.

In comparison to the perpendicular field geometry, transport in the presence of a parallel field has received relatively little attention. One of the earliest studies is that of Englert et al. 1 who observed a pronounced positive transverse magnetoresistance when an in-plane magnetic field was tilted slightly out of the plane of the 2DEG. This interesting observation was not understood at the time. More recently, the same effect was studied in considerably more detail by Leadley et al. 5 for a number of GaAs/AlGaAs heterojunctions. The explanation of this effect is the primary focus of our work.

A positive transverse magnetoresistance is known to occur in situations where conduction is provided by different kinds of carriers, for example, a heterojunction with two or more occupied subbands. In accord with classical theoryl, samples with only a single occupied subband do not show the effect (in fact, a negative magnetoresistance attributable to weak localization is usually observed). However the situation changes when the system is subjected to a parallel magnetic field which affects the electronic structure and leads to a field-induced anisotropy of the 2D Fermi contour. As noted by Leadley et al. analogous to the Fermi surface anisotropy found in metals and should similarly lead to a positive magnetoresistancet. Although the anisotropy of the Fermi contour is important, we 
shall see that it is not the only effect contributing to the positive magnetoresistance.

There have been a few theoretical considerations of this problem. The first calculation of transport in the presence of a parallel magnetic field was carried out by Tang and Butcher 6 . who considered a model in which the 2DEG is confined by a harmonic potential. The electronic states and energy dispersion can be obtained analytically for this model which simplifies the solution of the transport problem. They further assume that the electrons are scattered by short range $\delta$-function potentials and demonstrate within the framework of the Boltzmann transport theory that the conductivity of the electron gas is anisotropic with respect to the angle between the current and the parallel magnetic field. However they do not consider the additional effects of a perpendicular magnetic field. A similar model was used by Smrčka 10 in an attempt to explain the observed positive magnetoresistance. However his calculation invokes a dc conductivity in the third dimension perpendicular to the plane of the 2DEG which has no obvious physical meaning. More recently, Středa et al 11 developed a semiclassical Boltzmann transport theory of the magnetoresistance within a constant relaxation time approximation. The theory assumes that currents flowing in a direction perpendicular to the in-plane magnetic field induce a transverse Hall field perpendicular to the 2DEG as first suggested by Stern国. It is then argued that this field in turn leads to a correction to the in-plane conductivity which is the source of the positive magnetoresistance. However, it is unclear how this mechanism might account for the magnetoresistance when the current is flowing parallel to the in-plane field. In any event, since the argumentation is rather heuristic, a more rigorous explanation of the experimental observations is needed. We have therefore performed a more thorough calculation of the transport properties in a tilted magnetic field, utilizing a realistic model of both the heterojunction electronic structure and the scattering of electrons from remote ionized impurities. We shall demonstrate that the inherent anisotropy of these properties is ultimately the source of the positive transverse magnetoresistance. 
We perform our calculations for model heterojunctions which correspond to the G590 series of samples studied by Leadley et al. . $_{\text {By }}$ modelling these particular samples we hope to minimize any differences in electronic structure which might otherwise obscure a comparison between theory and experiment. The design characteristics of these samples have been dis-

cussed elsewhere 3 . The important variables are the areal acceptor and donor densities $\left(n_{A}\right.$ and $n_{D}$ ), which can be varied experimentally by means of the persistent photoconductivity effect. We shall deal specifically with two combinations, herein referred to as Sample 1 and Sample 2. Sample 1 has an acceptor density of $n_{A}=1.03 \times 10^{11} \mathrm{~cm}^{-2}$ and a donor density of $n_{D}=4.53 \times 10^{11} \mathrm{~cm}^{-2}$, giving a total electron density of $n_{T}=3.5 \times 10^{11} \mathrm{~cm}^{-2}$. It corresponds to the unilluminated experimental sample referred to as G590a which has only one occupied subband. For Sample 2, the areal densities are $n_{A}=0.1 \times 10^{11} \mathrm{~cm}^{-2}$ and $n_{D}=7.2 \times 10^{11} \mathrm{~cm}^{-2}$, resulting in a total electron density of $n_{T}=7.1 \times 10^{11} \mathrm{~cm}^{-2}$, corresponding to the experimental sample referred to as G590c. This sample is obtained from G590a by illumination and has two occupied subbands. A comparison of the theoretical and experimental zero-field mobilities provides one measure of how well we have been able to model these samples. We find $20.6 m^{2} / V s$ and $37.9 m^{2} / V s$ for Sample 1 and 2, respectively, which compare favourably with the experimental values of $17.4 \mathrm{~m}^{2} / \mathrm{Vs}$ and $94.0 \mathrm{~m}^{2} / \mathrm{Vs}$. We are therefore confident that our model heterojunctions closely represent the actual experimental samples.

\section{THEORY}

\section{A. Boltzmann Transport Theory}

In this section, we develop the Boltzmann transport equation for a 2DEG in the presence of a strong parallel magnetic field in the $x$-direction and a weak perpendicular component. The parallel field is taken into account quantum mechanically in the determination of the subband electronic structure (see Ref. 目). As a function of the in-plane wavevector $\mathbf{k}$, the dispersion of the subband energies $E_{n \mathbf{k}}$ is anisotropic and leads to noncircular constant 
energy contours. An example of this behaviour is shown in Figure 1, where the contours are illustrated for Sample 2 for two parallel magnetic fields, one $(B=5 T)$ for which two subbands are occupied and a second $(B=10 T)$ for which only a single subband is occupied.

The dynamics of the electrons in the presence of a perpendicular magnetic field component $B_{\perp}$ will be described semiclassically. The transport properties arising from an external electric field $\mathbf{E}$ are then determined using the Boltzmann equation

$$
\left.\frac{\partial f_{n}(\mathbf{k})}{\partial t}\right|_{s c a t}=-\left(\frac{e}{\hbar}\right) \mathbf{E} \cdot \nabla_{\mathbf{k}} f_{n}(\mathbf{k})-\frac{e}{\hbar c}\left(\mathbf{v}_{n \mathbf{k}} \times \mathbf{B}_{\perp}\right) \cdot \nabla_{\mathbf{k}} f_{n}(\mathbf{k})
$$

where $f_{n}(\mathbf{k})$ is the nonequilibrium distribution function of subband $n$ and $\mathbf{v}_{n \mathbf{k}}=\nabla_{\mathbf{k}} E_{n \mathbf{k}} / \hbar$ is the group velocity. Since the energy bands are neither parabolic nor isotropic in the presence of a parallel magnetic field, the electron velocity is not parallel to the wave vector k. Expressing $f_{n}(\mathbf{k})$ as

$$
f_{n}(\mathbf{k})=f^{0}\left(E_{n \mathbf{k}}\right)+g_{n}(\mathbf{k})
$$

where $f^{0}\left(E_{n \mathbf{k}}\right)$ is the equilibrium Fermi distribution and $g_{n}(\mathbf{k})$ is the deviation from equilibrium, the linearized Boltzmann equation is given by

$$
\left.\frac{\partial g_{n}(\mathbf{k})}{\partial t}\right|_{\text {scat }}=-\left(\frac{e}{\hbar}\right) \mathbf{E} \cdot \nabla_{\mathbf{k}} f^{0}\left(E_{n \mathbf{k}}\right)-\frac{e}{\hbar c}\left(\mathbf{v}_{n \mathbf{k}} \times \mathbf{B}_{\perp}\right) \cdot \nabla_{\mathbf{k}} g_{n}(\mathbf{k})
$$

The scattering term in (3) is

$$
\left.\frac{\partial g_{n}(\mathbf{k})}{\partial t}\right|_{s c a t}=\sum_{n^{\prime} \mathbf{k}^{\prime}}\left[g_{n^{\prime}}\left(\mathbf{k}^{\prime}\right)-g_{n}(\mathbf{k})\right] w_{n n^{\prime}}\left(\mathbf{k}, \mathbf{k}^{\prime}\right)
$$

where $w_{n n^{\prime}}\left(\mathbf{k}, \mathbf{k}^{\prime}\right)$ is the transition rate between the states $n \mathbf{k}$ and $n^{\prime} \mathbf{k}^{\prime}$. It satisfies the detailed balance condition $w_{n^{\prime} n}\left(\mathbf{k}^{\prime}, \mathbf{k}\right)=w_{n n^{\prime}}\left(\mathbf{k}, \mathbf{k}^{\prime}\right)$ which ensures the overall conservation of particle number.

In the following we shall assume that the scattering is due to impurities. In this case, Fermi's golden rule gives the transition probability

$$
w_{n n^{\prime}}\left(\mathbf{k}, \mathbf{k}^{\prime}\right)=\frac{2 \pi}{\hbar} \overline{\left|\left\langle n \mathbf{k}|V(\mathbf{r})| n^{\prime} \mathbf{k}^{\prime}\right\rangle\right|^{2}} \delta\left(E_{n \mathbf{k}}-E_{n^{\prime} \mathbf{k}^{\prime}}\right)
$$


where $V(\mathbf{r})$ is the screened impurity potential. The bar over the matrix element denotes an average over all possible impurity configurations. The important property for the present purposes is the energy-conserving delta function which corresponds to the elastic nature of the scattering. Making use of this dependence and defining the quantity $\xi_{n}(\mathbf{k})$ by

$$
g_{n}(\mathbf{k})=\frac{\partial f^{0}\left(E_{n \mathbf{k}}\right)}{\partial E_{n \mathbf{k}}} \xi_{n}(\mathbf{k})
$$

equation (3) can be reduced to

$$
\tau_{n}^{-1}(\mathbf{k}) \xi_{n}(\mathbf{k})=\sum_{n^{\prime} \mathbf{k}^{\prime}} w_{n n^{\prime}}\left(\mathbf{k}, \mathbf{k}^{\prime}\right) \xi_{n^{\prime}}\left(\mathbf{k}^{\prime}\right)+e \mathbf{E} \cdot \mathbf{v}_{n \mathbf{k}}+\frac{e}{\hbar c}\left(\mathbf{v}_{n \mathbf{k}} \times \mathbf{B}_{\perp}\right) \cdot \nabla_{\mathbf{k}} \xi_{n}(\mathbf{k})
$$

Here, $\tau_{n}(\mathbf{k})$ is the quantum lifetime

$$
\tau_{n}^{-1}(\mathbf{k})=\sum_{n^{\prime} \mathbf{k}^{\prime}} w_{n n^{\prime}}\left(\mathbf{k}, \mathbf{k}^{\prime}\right)
$$

which represents the total unweighted probability of scattering from the initial state $n \mathbf{k}$ to all available final states, including states in other subbands. Due to the more complicated energy subband structure in the presence of a parallel magnetic field, $w_{n n^{\prime}}\left(\mathbf{k}, \mathbf{k}^{\prime}\right)$ is not simply a function of $\mathbf{k}-\mathbf{k}^{\prime}$ and as a result, $\tau_{n}(\mathbf{k})$ is in general an anisotropic function of the wavevector $\mathbf{k}$.

The anisotropic energy band structure also complicates the solution of the Boltzmann equation. We find it useful to transform from the $\mathbf{k}$-space variables to a curvilinear coordinate system defined by the energy variable $\epsilon \equiv E_{n \mathbf{k}}$ and an angular variable $\phi$ corresponding to the orientation of the vector $\mathbf{k}$. To be specific, the position in $\mathbf{k}$-space of a state in the $n$th subband is referred to the position of the minimum of the subband energy which is displaced from $k_{y}=0$ because of the parallel magnetic field. This new origin is implicitly assumed in the following. The definition of these variables is illustrated in Figure 1. We have found that the use of the variable $\phi$ is more convenient in the present context than the phase angle $\theta$ which naturally appears in discussions of cyclotron motion (Ref. 6, Chap. 9).

With this variable transformation, equation (7) becomes

$$
\tau_{n}^{-1}(\epsilon, \phi) \xi_{n}(\epsilon, \phi)=\sum_{n^{\prime}} \frac{1}{2 \pi} \int_{0}^{2 \pi} d \phi J_{n^{\prime}}\left(\epsilon, \phi^{\prime}\right) P_{n n^{\prime}}\left(\epsilon, \phi, \phi^{\prime}\right) \xi_{n^{\prime}}\left(\epsilon, \phi^{\prime}\right)
$$




$$
+e \mathbf{E} \cdot \mathbf{v}_{n}(\epsilon, \phi)-\left(\frac{e B_{\perp}}{\hbar c}\right) v_{n}(\epsilon, \phi) \hat{\mathbf{t}}_{n} \cdot \nabla_{\mathbf{k}} \xi_{n}(\epsilon, \phi)
$$

where $\hat{\mathbf{t}}_{n}$ is a unit vector pointing in the direction of cyclotron motion on the constant energy contour of the $n$th subband at the point $\mathbf{k}$. The angular transition rate at energy $\epsilon$ appearing in (9) is defined by

$$
P_{n n^{\prime}}\left(E_{n \mathbf{k}}, \phi, \phi^{\prime}\right)=A \frac{m^{*}}{\hbar^{3}} \overline{\left|\left\langle n \mathbf{k}|V(\mathbf{r})| n^{\prime} \mathbf{k}^{\prime}\right\rangle\right|^{2}}
$$

in terms of which the quantum lifetime is given by

$$
\tau_{n}^{-1}(\epsilon, \phi)=\sum_{n^{\prime}} \frac{1}{2 \pi} \int_{0}^{2 \pi} d \phi^{\prime} J_{n^{\prime}}\left(\epsilon, \phi^{\prime}\right) P_{n n^{\prime}}\left(\epsilon, \phi, \phi^{\prime}\right) .
$$

The integrals in (9) and (11) contain the quantity

$$
J_{n}(\epsilon, \phi)=\frac{\hbar^{2} k}{m^{*}\left|\nabla_{\mathbf{k}} E_{n \mathbf{k}} \cdot \hat{\mathbf{k}}\right|}
$$

which is a dimensionless form of the Jacobian of the variable transformation. In this form it is just the ratio of the free particle velocity $\hbar k / m^{*}$ to the component of the actual velocity $\mathbf{v}_{n}(\mathbf{k})$ in the direction $\hat{\mathbf{k}}$. The deviation of this function from unity reflects the asymmetry of the constant energy contours in the presence of a parallel magnetic field.

The solution of (9) is easily obtained using a Fourier expansion in the angular variable, i.e.

$$
\xi_{n}(\epsilon, \phi)=\sum_{m} a_{n}^{(m)}(\epsilon) e^{i m \phi}
$$

Multiplying (9) by $e^{-i m \phi} J_{n}(\epsilon, \phi)$ and integrating over $\phi$ yields the set of linear equations

$$
\sum_{m^{\prime} n^{\prime}}\left[A_{n}^{\left(m-m^{\prime}\right)} \delta_{n n^{\prime}}-B_{n n^{\prime}}^{\left(m,-m^{\prime}\right)}+i m \omega_{c}^{0} \delta_{m m^{\prime}} \delta_{n n^{\prime}}\right] a_{n^{\prime}}^{\left(m^{\prime}\right)}=b_{n}^{(m)}
$$

with

$$
\begin{gathered}
A_{n}^{(m)}=\frac{1}{2 \pi} \int_{0}^{2 \pi} d \phi e^{-i m \phi} J_{n}(\phi) \tau_{n}^{-1}(\phi) \\
B_{n n^{\prime}}^{\left(m, m^{\prime}\right)}=\frac{1}{2 \pi} \int_{0}^{2 \pi} d \phi e^{-i m \phi} J_{n}(\phi) \frac{1}{2 \pi} \int_{0}^{2 \pi} d \phi^{\prime} e^{-i m^{\prime} \phi^{\prime}} J_{n^{\prime}}\left(\phi^{\prime}\right) P_{n n^{\prime}}\left(\phi, \phi^{\prime}\right)
\end{gathered}
$$


and

$$
b_{n}^{(m)}=\frac{1}{2 \pi} \int_{0}^{2 \pi} d \phi e^{-i m \phi} J_{n}(\phi) e \mathbf{E} \cdot \mathbf{v}_{n}(\phi)
$$

In these equations, the dependence on the energy variable $\epsilon$ has been suppressed. We note that the free electron cyclotron frequency $\omega_{c}^{0}=\frac{e B_{\perp}}{m^{*} c}$ appears in equation (14), and not the actual cyclotron frequency defined by $\omega_{c n}^{-1}=\frac{\hbar c}{e B_{\perp}} \oint \frac{d k}{v_{n}}$, where the line integral extends over a constant energy contour.

In the absence of a parallel magnetic field the energy dispersion is isotropic, $J_{n}(\phi)$ reduces to unity and the angular transition rate is only a function of the difference $\phi-\phi^{\prime}$. As a result, $\tau_{n}^{-1}(\phi)$ becomes independent of $\phi, b_{n}^{(m)}$ is proportional to $\delta_{m, \pm 1}$ and $a_{n}^{( \pm 1)}$ are the only non-vanishing expansion coefficients. In this limit, one recovers the usual form of the multisubband transport equations 13 .

In the present situation however, the equations defining the Fourier expansion coefficients are coupled. In practice, the expansion is truncated at some finite number of terms and the set of equations in (14) is solved with the inhomogeneous term taking on two possible values, one corresponding to an electric field in the $x$-direction and one in the $y$-direction. Once these solutions are known, the transport current density is obtained from

$$
\begin{aligned}
\mathbf{J} & =-\frac{2 e}{A} \sum_{n \mathbf{k}} g_{n}(\mathbf{k}) \mathbf{v}_{n \mathbf{k}} \\
& =\frac{m^{*} e}{2 \pi^{2} \hbar^{2}} \sum_{m n} \int_{-\infty}^{\infty} d \epsilon\left(-\frac{\partial f^{0}(\epsilon)}{\partial \epsilon}\right) a_{n}^{(m)}(\epsilon) \int_{0}^{2 \pi} d \phi J_{n}(\epsilon, \phi) \mathbf{v}_{n}(\epsilon, \phi) e^{i m \phi}
\end{aligned}
$$

Since the Fourier coefficient $a_{n}^{(m)}$ depends linearly on the electric field, we can define the conductivity tensor as

$$
\sigma_{\mu \nu}=\frac{m^{*} e}{2 \pi^{2} \hbar^{2}} \sum_{m n} \int_{-\infty}^{\infty} d \epsilon\left(-\frac{\partial f^{0}(\epsilon)}{\partial \epsilon}\right) \frac{\partial a_{n}^{(m)}(\epsilon)}{\partial E_{\nu}} \int_{0}^{2 \pi} d \phi J_{n}(\epsilon, \phi) v_{n \mu}(\epsilon, \phi) e^{i m \phi}
$$

In the $B_{\perp} \rightarrow 0$ limit, one can show that $\sigma_{\mu \nu}$ is diagonal, but $\sigma_{x x} \neq \sigma_{y y}$. As a result of the distortion of the Fermi contour, the current flow is not parallel to the applied electric field except for the special cases when the electric field is either parallel or perpendicular to the in-plane magnetic field. 
We finally comment on whether or not the transverse Hall field proposed by Středa et al.11 is a possible mechanism for the positive magnetoresistance. The nonequilibrium distribution function in (2) implies a spatial redistribution of the electronic charge in a direction normal to the interface given by

$$
\delta n(z)=2 \sum_{n \mathbf{k}} g_{n}(\mathbf{k})\left|\phi_{n k_{y}}(z)\right|^{2}
$$

where $\phi_{n k_{y}}(z)$ is the subband wavefunction in the presence of the in-plane magnetic field. This redistribution gives rise to a transverse Hall field and a Hall potential which is estimated to be of the order of microvolts under typical current carrying conditions. 12,9 These potentials

are small on the energy scale of the confining potential 14 and will lead to small changes in the energy band dispersion. Corrections to the Boltzmann equation (for example, as a result of changes in the electron velocity) are therefore of higher order in the applied electric field and, at the level of the linearized Boltzmann theory, can be safely neglected. A transverse Hall field is therefore not relevant to in-plane transport.

\section{B. Evaluation of the Scattering Elements $P_{n n^{\prime}}\left(\mathbf{k}, \mathbf{k}^{\prime}\right)$}

We now turn our attention to the calculation of $P_{n n^{\prime}}\left(\mathbf{k}, \mathbf{k}^{\prime}\right)$ in $(\sqrt{10})$ for a situation in which scattering is due to remote ionized impurities. Using the form of the subband states in the presence of a parallel magnetic field, the potential matrix element has the form

$$
\left\langle n \mathbf{k}|V(\mathbf{r})| n^{\prime} \mathbf{k}^{\prime}\right\rangle=\frac{1}{A} \int d z \phi_{n k_{y}}(z) \phi_{n^{\prime} k_{y}^{\prime}}(z) V(z, \mathbf{q})
$$

where $\mathbf{q}=\mathbf{k}-\mathbf{k}^{\prime}$ and

$$
V(z, \mathbf{q})=\int d \mathbf{s} e^{-i \mathbf{q} \cdot \mathbf{s}} V(\mathbf{s}, z)
$$

is the 2D Fourier transform of the scattering potential. We note that the evaluation of (21) for the parallel field configuration is considerably more involved because of the $k_{y}$-dependence of the subband states. As a result, the potential matrix elements do not simply depend on the momentum transfer $\mathbf{q}$ which appears in the Fourier transform of the impurity potential. 
We assume that the impurity potential arises from a distribution of remote ionized impurities located at sites $\left(\mathbf{R}_{i}, Z_{i}\right)$. These impurities (of charge $e$ ) give rise to the bare electrostatic potential

$$
\begin{aligned}
\phi^{e x t}(z, \mathbf{q}) & =\sum_{i}\left(\frac{2 \pi e}{\kappa q}\right) e^{-q z} e^{q Z_{i}} e^{-i \mathbf{q} \cdot \mathbf{R}_{i}} \\
& \equiv\left(\frac{2 \pi e}{\kappa q}\right) I(\mathbf{q}) e^{-q z}
\end{aligned}
$$

where a two dimensional Fourier transform has been taken in the plane of the 2DEG and we have assumed that the position of the charged impurities is such that $Z_{i}<z$. $\kappa$ is the dielectric constant of the material in which the 2DEG is imbedded. The quantity $I(\mathbf{q})$ determines the amplitude of the exponentially decaying Fourier transform and contains all information regarding the spatial distribution of the ionized donor impurities.

The external impurity potential is screened by the 2DEG and it is the final screened potential which is responsible for the electron scattering. We shall account for the screening at the level of the random phase approximation in which the electrons respond self-consistently to the ionized impurity potential. Defining $\chi^{0}\left(z, z^{\prime}, \mathbf{q}\right)$ to be the $2 \mathrm{D}$ Fourier transform of the independent particle density response function, the change in electron density due to the impurities is given by

$$
\delta n(z, \mathbf{q})=e \int d z^{\prime} \chi^{0}\left(z, z^{\prime}, \mathbf{q}\right) \phi^{t o t}\left(z^{\prime}, \mathbf{q}\right)
$$

where $\phi^{\text {tot }}(z, \mathbf{q})$ is the total electrostatic potential acting on the electrons. This potential is given by

$$
\phi^{t o t}(z, \mathbf{q})=\phi^{e x t}(z, \mathbf{q})+\phi^{i n d}(z, \mathbf{q})
$$

where the second term is the induced electrostatic potential

$$
\phi^{i n d}(z, \mathbf{q})=-\left(\frac{2 \pi e}{\kappa q}\right) \int d z^{\prime} e^{-q\left|z-z^{\prime}\right|} \delta n\left(z^{\prime}, \mathbf{q}\right)
$$

arising from the induced electron charge density. 
In the absence of a parallel magnetic field, the subband envelope functions are $\mathbf{k}$ independent and the calculation of the density response function is relatively straightforward. It takes the form

$$
\chi^{0}\left(z, z^{\prime}, \mathbf{q}\right)=\sum_{\beta} f_{\beta}(z) f_{\beta}\left(z^{\prime}\right) \chi_{\beta}^{0}(q)
$$

where the index $\beta$ represents a pair of subband indices $\left(n, n^{\prime}\right)$ and $f_{\beta}(z)$ is the product $\phi_{n}(z) \phi_{n^{\prime}}(z)$ of two subband envelope functions. The factor $\chi_{\beta}^{0}(q)$ is just the intersubband response function for an ideal 2DEG which depends on the subband structure through the subband energy levels and the position of the Fermi level. Analytic expressions for $\chi_{\beta}^{0}(q)$ are available (see, for example, Ref. 15).

Once a parallel magnetic field is present, the subband states acquire a non-trivial $k_{y}$ dependence and $\chi^{0}\left(z, z^{\prime}, \mathbf{q}\right)$ cannot be obtained analytically. To avoid an excessive and largely unnecessary amount of numerical work, we shall make a simple approximation motivated by the following observation. Although the individual subband states are strongly modified by the parallel field, depending on the value of $k_{y}$, the overall electron density distribution is relatively insensitive to the field. This is illustrated for Sample 2 in Figure 2, where we compare the ground state density for $B_{\|}=0 T$ to the density at the relatively high field of $B_{\|}=10 T$. As can be seen, the effect of the field is minor, even for this case in which the second occupied subband is depopulated by the magnetic field. One would therefore expect the screening of the impurity potential to take place in the presence of the parallel field in much the same way as in the zero-field limit. We therefore adopt the physically reasonable approximation of screening the impurities by the zero-field response function given by (27).

However, one complication must be addressed: higher occupied subbands will depopulate with increasing parallel magnetic field. Since different subbands screen differently, maintaining the zero field populations in the calculation of $\chi^{0}\left(z, z^{\prime}, \mathbf{q}\right)$ introduces an error which can be avoided in the following way. The subband populations $n_{i}(B)$ are first determined from a fully self-consistent electronic structure calculation which is known to reproduce the observed field dependence quite accurately. 1 We then make use of this in- 
formation in the calculation of $\chi_{\beta}^{0}(q)$ by simply defining fictitious subband energies $E_{i}^{0}$ to ensure that the subband densities are given correctly, that is,

$$
2 \pi n_{i}\left(B_{\|}\right)=E_{F}-E_{i}^{0}
$$

This is sufficient to define the intersubband response function for the occupied subbands. In the cases we have dealt with, a maximum of two subbands are occupied and we have therefore truncated $\chi_{\beta}^{0}(q)$ to a two-by-two matrix corresponding to the lowest two subbands. Once the second subband is depopulated, the second subband still contributes to the screening by providing final states to which the first subband electrons can be excited. In this situation the second subband is positioned relative to the Fermi level according to the self-consistent calculation, while the first subband energy is still determined by (28). In practice we have found that these refinements have only a slight effect on the calculated scattering matrix elements since the lowest subband holds most of the electrons and contributes most of the screening. Nevertheless, since relatively little additional effort is required to determine these corrections, we have retained them in all of the screening calculations.

With this prescription for $\chi_{\beta}^{0}(q)$ and $\chi^{0}\left(z, z^{\prime}, \mathbf{q}\right)$, the screened impurity potential is obtained from the set of equations (23)-(26). Expressing $\phi^{\text {tot }}(\mathbf{q}, z)$ as

$$
\phi^{t o t}(z, \mathbf{q})=\left(\frac{2 \pi e}{\kappa q}\right) I(\mathbf{q}) J(z, q)
$$

we have

$$
J(z, q)=e^{-q z}-\left(\frac{2 \pi e^{2}}{\kappa q}\right) \sum_{\beta} \chi_{\beta}^{0}(q) \int d z^{\prime} \int d z^{\prime \prime} e^{-q\left|z-z^{\prime}\right|} f_{\beta}\left(z^{\prime}\right) f_{\beta}\left(z^{\prime \prime}\right) J\left(z^{\prime \prime}, q\right) .
$$

We note that this integral equation has a separable kernel and it can therefore be reduced to a matrix problem. Multiplying (30) by $f_{\alpha}(z)$ and integrating over $z$ gives

$$
J_{\alpha}(q)=\int d z f_{\alpha}(z) e^{-q z}-\sum_{\beta} F_{\alpha \beta}(q) \chi_{\beta}^{0}(q) J_{\beta}(q)
$$

where

$$
J_{\alpha}(q)=\int d z f_{\alpha}(z) J(z, q)
$$


and

$$
F_{\alpha \beta}(q)=\left(\frac{2 \pi e^{2}}{\kappa q}\right) \int d z \int d z^{\prime} f_{\alpha}(z) f_{\beta}\left(z^{\prime}\right) e^{-q\left|z-z^{\prime}\right|}
$$

is a Coulomb form factor. As mentioned earlier, we retain two subbands in our calculations, so that the screening effect of the second subband is still present even when it is depopulated.

The solution of (31) is substituted into (30) to obtain the $z$-dependence of the screened impurity potential required in the calculation of the scattering matrix elements. In particular, the angular transition rate takes the form

$$
P_{n n^{\prime}}\left(\mathbf{k}, \mathbf{k}^{\prime}\right)=\frac{m^{*}}{\hbar^{3}}\left|\left(\frac{2 \pi e^{2}}{\kappa q}\right) \int d z \phi_{n k_{y}}(z) \phi_{n^{\prime} k_{y}^{\prime}}(z) J(z, q)\right|^{2} \frac{1}{A} \overline{|I(\mathbf{q})|^{2}}
$$

where the bar over the final factor denotes a configuration average. For uncorrelated impurity positions, we have

$$
\frac{1}{A} \overline{|I(\mathbf{q})|^{2}}=\int d z \rho_{i}(z) e^{2 q z}
$$

where $\rho_{i}(z)$ is the average spatial density of ionized impurities. For the special case of a delta-doped layer with $\rho_{i}(z)=n_{d} \delta(z+s)$, (35) becomes $n_{d} e^{-2 q s}$, showing that the effect of the impurities diminishes exponentially with the setback distance $s$.

We can compare this result with the idealized situation of short range impurity scattering

considered by Tang and Butcher $\mathrm{B}$. For a sheet of $\delta$-function scatterers located in the plane $z=z_{0}$, we find $P_{n n^{\prime}}\left(\mathbf{k}, \mathbf{k}^{\prime}\right) \propto\left|\phi_{n k_{y}}\left(z_{0}\right)\right|^{2}\left|\phi_{n^{\prime} k_{y}^{\prime}}\left(z_{0}\right)\right|^{2}$, which must be integrated over $z_{0}$ to correspond to a uniform distribution of scatterers throughout the region of the 2DEG. This scattering rate is independent of $k_{x}$ and $k_{x}^{\prime}$ which, as noted by Tang and Butcher 8 , simplifies the solution of the Boltzmann equation in the case of $\mathbf{E} \| \mathbf{B}_{\|}$. For the more realistic situation of remote charged impurities this simplification does not arise and the $\mathbf{E} \| \mathbf{B}_{\|}$case requires a treatment similar to that of the $\mathbf{E} \perp \mathbf{B}_{\|}$case.

\section{RESULTS AND DISCUSSION}

All of the interesting magnetotransport effects in the presence of an in-plane magnetic field ultimately arise from the distortion of the Fermi contours illustrated in Figure 1 and 
the associated behaviour of the subband wave functions. That this could lead to a positive transverse magnetoresistance was already appreciated by Leadley et al. who viewed the electrons on the distorted Fermi contour as different kinds of carriers having different mobilities. The usual treatment of parallel transport 6 would then give rise to a positive magnetoresistance. They attributed variations in the mobility to a k-dependent effective mass which is due to the altered band structure. However, within a Boltzmann transport theory it is the electron velocity which emerges as the important dynamical variable, and the effective mass appears only when the energy dispersion is strictly parabolic, which is not the case in the present situation. In any event, a much more significant factor is the variation of the electronic scattering rates around the Fermi contour. In the following we shall try to differentiate between the kinematic effects which arise from the energy band structure and the dynamical effects associated with impurity scattering.

Figure 1 shows an example of the Fermi contour anisotropy for a sample in which two subbands are occupied at zero field. As can be seen, the distortion of the Fermi contour of the first subband increases with parallel field strength and eventually takes on the shape of an egg. As this distortion is developing, the energy separation between the first and second subbands increases and leads to the depopulation of the second subband. (For Sample 2, complete depopulation occurs at approximately $B_{\|} \sim 5.6 T$.) Interestingly, the minimum in the second subband also displaces relative to that in the first, so that the pocket of second subband states approaches the first subband Fermi contour with increasing field. This too will be seen to have an important effect. However, we shall begin by considering the simpler situation in which only a single subband is occupied, either because the electron density is low or because the field is sufficiently high to have depopulated the higher subband. Since our calculations are done for zero temperature, only the states at the Fermi energy are relevant and we can restrict the solution of (9) to $\epsilon=E_{F}$. It should be understood that all quantities are calculated at this energy.

One measure of the Fermi contour anisotropy is the Jacobian defined in (12). In Figure 3 we plot the inverse of this quantity as a function of the angular position around the Fermi 
contour, together with the ratio of the magnitude of the velocity to the free-electron velocity $\hbar k / m^{*}$. We recall that the $\mathbf{k}$-vector in this context is defined with respect to the position of the subband minimum and not the more usual $\mathbf{k}$-space origin. One consequence of this definition is that there is only a small difference between the curves in Figure 3, indicating that the normal to the Fermi contour does not deviate much from the direction of $\mathbf{k}$. In this respect, the Fermi contour is still rather circular in nature, despite its visual appearance. On the other hand, the four-fold variation of $J_{1}^{-1}(\phi)$ around the contour is showing that the modified energy dispersion is having a dramatic effect on the electron velocity. There are two effects coming into play. The first is the overall elongation along the $k_{y}$-axis which is a result of a magnetic field enhancement of the effective mass in this direction. As discussed previously $\mathbb{1}$, this leads to an enhancement of the electronic density of states above the ideal $2 \mathrm{D}$ value. It is this effect that accounts for the decrease in $J_{1}^{-1}(\phi)$ near $\phi=\pi$. However this same effect is swamped near $\phi=0$ by a much larger reduction of the velocity coming from the flattening of the energy bands. Only at $\phi=\pi / 2$ is there no effect of the Fermi contour anisotropy, as the dependence of the energy on $k_{x}$ is unchanged by the parallel magnetic field.

The difference in behaviour of the energy dispersion near $\phi=0$ and $\phi=\pi$ is a reflection of the $k_{y}$-dependence of the electron wavefunctions. Electrons with $k_{y}$ negative $(\phi \rightarrow \pi)$ experience a magnetic potential which drives them into the interface, whereas states with positive $k_{y}(\phi \rightarrow 0)$ are pushed away from the interface. The latter is a stronger effect, since the confining potential in a direction away from the interface is much softer, particularly when the background acceptor density is low. These differences are illustrated in Figure 4 which shows the centroid of the subband probability densities as a function of $k_{y}$. One can see that there is only a slight change in the centroid position as the wavefunctions are driven into the interface $(\pi / 2<\phi<\pi)$, but there is a much larger variation for those states being pushed in the opposite direction $(0<\phi<\pi / 2)$.

The change in mean position has a dramatic effect on the angular scattering rate (34). It is particularly sensitive to the wavefunction position since the bare scattering potential is 
an exponential function of the distance from the impurity layer. Furthermore, those states which are further from the interface experience the full screening effect of the 2DEG which is interposed between them and the ionized donor impurities. The angular scattering rate depends on the two angular variables $\phi$ and $\phi^{\prime}$ which specify the orientation of the initial and final wavevectors, respectively. Part of this angular dependence arises from the impurity form factor $\overline{|I(\mathbf{q})|^{2}}$ which depends on the momentum transfer $\mathbf{q}=\mathbf{k}^{\prime}-\mathbf{k}$. This factor is common to the zero field limit and we therefore choose to plot a normalized transition rate which has this factor removed in order to isolate the new effects associated with the dependence of the scattering matrix elements on the subband states. In particular, for the case of a single subband we consider the quantity

$$
\bar{P}_{11}(\phi, \Delta \phi)=\frac{A}{\overline{|I(\mathbf{q})|^{2}}} P_{11}(\phi, \phi+\Delta \phi)
$$

where $\Delta \phi$ is the angle through which the electron starting at the point $\phi$ is scattered. Because of the anisotropy of the Fermi contour, $P_{11}(\phi, \phi+\Delta \phi)$ is not a symmetric function of the scattering angle, except at the special points $\phi=0$ and $\phi=\pi$. More generally, we have the symmetry $P_{11}\left(\phi, \phi^{\prime}\right)=P_{11}\left(2 \pi-\phi, 2 \pi-\phi^{\prime}\right)$.

Figure 5 shows the normalized scattering rate as a function of the starting angle $\phi$ for several scattering angles. For a given scattering angle $\Delta \phi$, the momentum transfer $\mathbf{q}$ is approximately constant and the dependence on $\phi$ is an indication of the strong anisotropy arising mainly from the $k_{y}$-dependence of the subband wavefunctions. The curve for $\Delta \phi=0$ corresponds to the limit of small angle scattering and shows a strong maximum at $\phi=\pi$. At this angle, the subband states are closest to the impurities where the screened potential $J(z, q)$ is relatively large. As $\phi \rightarrow 0$ (or $2 \pi$ ), the subband states move away from the impurities and the scattering rate diminishes accordingly. A similar behaviour is seen for the other scattering angles, although now an asymmetry with respect to $\pm \Delta \phi$ is evident. The decreasing magnitude of the scattering rate with increasing scattering angle is partly due to the momentum transfer dependence of the screened potential $J(z, q)$ which decreases with increasing $q$. 
It is also of interest to consider the anisotropy of the quantum lifetime defined in (8). Given that the scattering rate drops off rapidly with increasing momentum transfer q, the inverse quantum lifetime is dominated by small angle scattering. In Figure 6 the anisotropy of the quantum lifetime is illustrated for Sample 1 at a field of $10 T$. The lifetime shows a four-fold variation between its maximum at $\phi=0$ and its minimum at $\phi=\pi$, the latter occurring when the angular scattering rate has its maximum, as shown in Figure 5. To isolate the effects of the shape of the Fermi contour itself, we can perform a model calculation in which the actual subband wavefunctions are replaced by their zero-field limit. The lifetime anisotropy in this case is shown by the dashed line in Figure 6. Since the angular scattering rate in (34) is now only a function of the momentum transfer, its anisotropy is relatively weak and the lifetime anisotropy is dominated by the Jacobian factor in (11). As a result, the dashed curve in Figure 6 mimics the behaviour of $J_{1}^{-1}(\phi)$ in Figure 3. It is clear from a comparison of the two curves in Figure 6 that the full lifetime anisotropy is coming mainly from the magnetic field dependence of the subband wavefunctions. The variation with parallel magnetic field strength is shown in Figure 7. The lifetime at zero field is of course isotropic and the anisotropy about this value is seen to increase approximately in proportion to the parallel field.

To illustrate the effect of multiple subband occupancy, we have performed similar lifetime calculations for Sample 2 at an intermediate field (5T) where both subbands are still occupied. In this instance, we can define multi-band quantum lifetimes by the equation

$$
\tau_{n n^{\prime}}^{-1}(\phi)=\tau_{n n^{\prime}}^{-1}(\mathbf{k})=\sum_{\mathbf{k}^{\prime}} w_{n n^{\prime}}\left(\mathbf{k}, \mathbf{k}^{\prime}\right)
$$

These lifetimes represent the unweighted probability of an electron scattering from the state $\mathbf{k}(\phi)$ in subband $n$ to all other states in subband $n^{\prime}$. The two-band quantum lifetimes presented in Figure 8 can be seen to exhibit quite different anisotropies. For $\tau_{11}$, we basically have the same behaviour shown in Figure 7 for the single-subband sample, but the magnitude of the anisotropy is significantly larger because of the smaller acceptor density in this sample. $\tau_{22}$ has a similar behaviour, although the anisotropy is reduced because of the smaller range 
of $k_{y}$ values in the second subband. For $\tau_{21}$ we see a slight increase in the lifetime as $\phi \rightarrow \pi$. This arises since the second subband states near $\phi=\pi$ tend to experience a slightly larger average momentum transfer when scattering into the first subband than do the states near $\phi=0$. However, the anisotropy of this intersubband lifetime is again small due to the small size of the pocket of second subband states. The variation of $\tau_{12}$ is much more dramatic, covering almost three orders of magnitude. This intersubband lifetime shows a pronounced minimum near $\phi \sim \pi / 8$ which is the region where the first Fermi contour comes closest to the second subband. The reduced average momentum transfer for these states results in a scattering 'hot-spot' at which the lifetime is relatively small.

In the zero field limit, the single subband transport lifetime differs from the quantum lifetime by the appearance of an additional $(1-\cos \phi)$ weighting factor in the integrand of (8). In the presence of a parallel magnetic field, there unfortunately is no similar expression which can be used to define a transport lifetime. Instead, one must deal directly with the non-equilibrium distribution function as determined by the solution of the Boltzmann equation. This distribution function can always be expressed in the form

$$
\xi_{n}(\phi) \equiv \mathbf{E} \cdot \vec{\Lambda}_{n}(\phi)
$$

which introduces the vector mean-free-path $\vec{\Lambda}_{n}(\phi)$. In general, the mean-free-path is not parallel to the velocity vector at the point $\phi$ and for this reason the conventional definition of a transport lifetime is not appropriate. Nevertheless, for a given direction of the electric field, it is possible to parameterize the mean-free-path in terms of a lifetime. For example, for $\mathbf{E}=E \hat{\mathbf{x}}$ (i.e., parallel to the in-plane magnetic field) we can write

$$
\Lambda_{n x}(\phi) \equiv v_{n x}(\phi) \tau_{n x}^{t r}(\phi) .
$$

Since the zeroes of $\xi_{n}(\phi)$ coincide by symmetry with those of $v_{n x}(\phi)$ when the electric field is in the $x$-direction, $\tau_{n x}^{t r}(\phi)$ is a well-defined quantity. This is not the case if the electric field is oriented in the $y$-direction and a similarly defined lifetime would exhibit singularities at certain points on the Fermi contour which have no physical significance. We shall therefore 
simply use $\tau_{n x}^{t r}(\phi)$ as a convenient parameterization in order to visualize the anisotropy of the solution to the Boltzmann equation.

Figure 9 shows the transport lifetime of Sample 1 defined according to (39), normalized by the zero field transport lifetime, at three magnetic fields. For lower fields we see a monotonic angular variation similar to that of the quantum lifetime, with those states at $\phi \rightarrow 0$ having a 50 percent greater lifetime than those at $\phi \rightarrow \pi$. The states near $\phi=\pi / 2$ which contribute the most to the current have a lifetime very similar to the zero field lifetime. As the field strength increases, the transport anisotropy changes in character, with additional structure appearing near $\phi=0$. More importantly, the average lifetime decreases with increasing field and results in a positive parallel field magnetoresistance. This effect is illustrated in Figure 10 which shows the magnetoresistance for the current parallel and perpendicular to the in-plane magnetic field. The magnetoresistance is approximately parabolic and of a sizable magnitude for field strengths of the order of 10T. In addition, we see that the magnetoresistance is larger for $\mathbf{J} \perp \mathbf{B}_{\|}$than for $\mathbf{J} \| \mathbf{B}_{\|}$, in qualitative agreement with experiment11, although it should be noted that the experimental anisotropy seems to vary from sample to sample in an unpredictable way.

We finally consider the transverse magnetoresistance which arises with a perpendicular component of the magnetic field. This component induces a cyclotron motion of the electrons around the Fermi contour and leads to an averaging of the anisotropic scattering rates. Figure 11 illustrates the behaviour found for a series of parallel magnetic field strengths for a situation in which two subbands are initially occupied. The model parameters in this case are $n_{A}=0.3 \times 10^{11} \mathrm{~cm}^{-2}$ and $n_{D}=7.4 \times 10^{11} \mathrm{~cm}^{-2}\left(n_{T}\right.$ is still $\left.7.1 \times 10^{11} \mathrm{~cm}^{-2}\right)$ instead of the values $n_{A}=0.1 \times 10^{11} \mathrm{~cm}^{-2}$ and $n_{D}=7.2 \times 10^{11} \mathrm{~cm}^{-2}$ used for Sample 2. This adjustment was made since the two subband mobilities for Sample 2 are almost identical and no positive magnetoresistance is found for zero parallel field. By increasing the acceptor concentration the calculated magnetoresistance correponds more closely to that observed experimentally and a more meaningful comparison of the parallel field dependence can be made. In addition, with the adjusted parameters the second subband depopulates at approximately $4 T$, which 
is in better agreement with the G590c sample of Leadley et al.0

The $B_{\|}=0 T$ curve in Figure 11 is the usual positive magnetoresistance associated with the different carriers in the two subbands (although it should be stressed that the carriers are not independent since intersubband scattering is included ${ }^{3}$ ). As the parallel magnetic field is increased, both the depth of the magnetoresistance dip, and the field at which saturation occurs, increase. This is in good qualitative agreement with the observations of Leadley et al.5 on their G590c sample, although the saturation field we find is approximately twice the value observed. Since saturation occurs when $\omega_{c} \tau \simeq 1$, the discrepancy can be explained in terms of the difference between our calculated mobility of $\mu=37.7 \mathrm{~m}^{2} / \mathrm{Vs}$ and the experimental value of $94.0 \mathrm{~m}^{2} / \mathrm{Vs}$. Once the depopulation field of about $4 T$ is exceeded, we find that the saturation field decreases suddenly to a value below the zero parallel field value due to the elimination of intersubband scattering and then remains constant, in marked contrast to the behaviour found below the depopulation field. The increase of the magnetoresistance with increasing parallel field in the single-subband limit is simply a consequence of the increasing lifetime anisotropy shown in Figure 7. This behaviour is in excellent qualitative agreement with that observed.

In Figure 12 we give an example of the kind of anisotropy that could be expected in the perpendicular field magnetoresistance. Figure (a) is for the same sample discussed in Figure 10 and shows a magnetoresistance which is only slightly larger for $\mathbf{J} \| \mathbf{B}_{\|}$than for $\mathbf{J} \perp \mathbf{B}_{\|}$. Figure (b), corresponding to the $B_{\|}=5 T$ curve in Figure 11, shows a much larger anisotropy with the magnetoresistance still being larger for $\mathbf{J} \| \mathbf{B}_{\|}$than for $\mathbf{J} \perp$ $\mathbf{B}_{\|}$. This relative magnitude of the two magnetoresistances persists over a range of sample parameters and is opposite to the relative magnitude oberved in one particular sample.11 We have no explanantion for this difference. It should also be noted that the experimental magnetoresistance tends not to saturate, but passes through a maximum before falling at higher magnetic fields.

To further quantify our results we show in Figure 13 the normalized magnetoresistance 


$$
\frac{\Delta \rho_{x x}}{\rho_{0}}=\frac{\rho_{x x}\left(B_{\perp}=\infty\right)-\rho_{x x}\left(B_{\perp}=0 T\right)}{\rho_{x x}\left(B_{\perp}=0 T\right)}
$$

for a range of acceptor densities with the total electron density fixed at $n_{T}=7.1 \times 10^{11} \mathrm{~cm}^{-2}$. A decrease in $n_{A}$ can be induced by illumination. In agreement with observation, the magnetoresistance increases with decreasing $n_{A}$; Figure 14(a) shows this dependence in a different way. This variation reflects the effect of illumination on the confining potential. As $n_{A}$ decreases, the confining potential becomes softer and the subband states are more strongly affected by a parallel magnetic field. In fact, we have found that $\Delta \rho_{x x} / \rho_{0}$ is far more sensitive to variations in $n_{A}$ than in any of the other material parameters. For example, Figure 14(b) illustrates the effects of varying the total electron density $n_{T}$ while keeping the parallel magnetic field constant. For low values of $n_{A}$, an increase in the total electron density translates into a reduction of the anisotropy in the system and a lower $\Delta \rho_{x x} / \rho_{0}$. This is mainly due to an increase in the confinement of the self-consistent heterostructure potential with increasing $n_{T}$. For higher values of $n_{A}$, the acceptors are themselves providing a stronger confining potential, and the effect of varying $n_{T}$ is greatly reduced.

A comparison of our results in Figure 13 with the experimental data of Leadley et al. permits an estimate of the acceptor densities in the experimental samples. We find that $n_{A} \simeq$ $0.35 \times 10^{11} \mathrm{~cm}^{-2}$ is appropriate for the illuminated sample (G590c), while $n_{A} \simeq 1.2-1.4 \times$ $10^{11} \mathrm{~cm}^{-2}$ for the dark sample (G590a). These values indicate that the change in acceptor

density as a result of illumination is $\Delta n_{A} \simeq 1 \times 10^{11} \mathrm{~cm}^{-2}$, which is consistent with the previously determined value 3 . Interestingly, our estimates suggest that a significant residual acceptor density remains after illumination, in contrast to what is sometimes assumed. If the acceptor density were eliminated completely, our calculations would yield a much larger magnetoresistance than observed.

\section{CONCLUSIONS}

We have performed extensive calculations of the transport properties of a 2DEG in the presence of parallel and perpendicular magnetic field components. The in-plane component 
leads to a significant perturbation of the electronic structure which manifests itself as a distortion of the Fermi contours and a magnetic field depopulation of higher lying subbands. These features are one source of the anisotropy that emerges in both the quantum and transport lifetimes. We have also analyzed the impact of the parallel magnetic field on the scattering of electrons from the long-range potential due to remote ionized donors. The scattering at different points on the Fermi contour is found to be highly anisotropic as a result of the field dependence of the subband wavefunctions. This detailed scattering information is then used in a Boltzmann transport theory which treats the perpendicular field component semiclassically. Our general solution of the Boltzmann transport equation includes fully the effects of the electronic structure, intersubband scattering and the anisotropy of the nonequilibrium distribution function. Application of the theory to a realistic heterojunction is found to yield results which are in good qualitative agreement with experiment. In particular, our calculations account for the observed positive transverse magnetoresistance and its detailed dependence on the magnitude of the in-plane magnetic field in both the one- and two-subband regimes. It is clear from our calculations that a careful treatment of the electronic structure, long-range impurity scattering and transport behaviour are all needed to obtain a complete understanding of the experimental results.

\section{ACKNOWLEDEGMENTS}

This work was partially supported by grants from the Natural Sciences and Engineering Research Council (NSERC) of Canada. 


\section{REFERENCES}

${ }^{1}$ J.M. Heisz and E. Zaremba, Semicond. Sci. Technol. 8, 575 (1993).

${ }^{2}$ T. Jungwirth and L. Smrčka, J. Phys.: Condens. Matter 5, L217(1993).

${ }^{3}$ R. Fletcher, J.J. Harris and C.T. Foxon, Semicond. Sci. Technol. 6, 54 (1991).

${ }^{4}$ Th. Englert, J.C. Maan, D.C. Tsui and A.C. Gossard, Solid State Commun. 45, 989 (1983).

${ }^{5}$ D.R. Leadley, R.J. Nicholas, J.J. Harris and C.T. Foxon, 20th International Conference on Physics of Semiconductors, Ed. E.M. Anastassakis and J.D. Joannopoulos (World Scientific, Singapore), 1609 (1990).

${ }^{6}$ J. M. Ziman, Principles of the Theory of Solids (Cambridge University Press, London, 1964), Chap. 7.

${ }^{7}$ A.B. Pippard, The Physics of Metals, Ed. J. M. Ziman 1, 165 (1969).

${ }^{8}$ H. Tang and P. N. Butcher, J. Phys. C: Solid State Phys. 21, 3313 (1988).

${ }^{9}$ H. Tang and P. N. Butcher, J. Phys. C: Solid State Phys. 21, 3959 (1988).

${ }^{10}$ L. Smrčka, Hel. Phys. Act. 65, 355 (1992).

${ }^{11}$ P. Středa, P. Vašek and M. Cukr, Phys. Rev. B51, 11144 (1995).

${ }^{12}$ F. Stern, Phys. Rev. Lett. 21, 1687 (1968).

${ }^{13}$ E. Zaremba, Phys. Rev. B45, 14143 (1992).

14 The redistribution of the electronic charge must in principle be determined selfconsistently, but this does not alter the argument that the changes are proportional to the applied electric field.

${ }^{15}$ R. Fletcher, E. Zaremba, M. D’Iorio, C. T. Foxon and J. J. Harris, Phys. Rev. B41, 10649 (1990). 


\section{FIGURES}

FIG. 1. Constant Fermi energy contours for Sample 2 with a parallel magnetic field of (a) $5 T$ (two-band occupancy) and (b) $10 T$ (single-band occupancy). $k_{x}$ and $k_{y}$ are in units of inverse Bohr radii $\left(\left(a^{*}\right)^{-1}\right)$. The angular variable $\phi$ defines the position on the Fermi contour with respect to the position of the first subband minimum, $k_{1}^{\text {min }}$; a similar angular coordinate is defined for the second subband, but is not shown.

FIG. 2. The spatial density profile of the 2DEG in Sample 2 as a function of distance from the interface, for zero magnetic field (solid line) and for a parallel magnetic field of $10 T$ (dashed line).

FIG. 3. The inverse Jacobian $m^{*} \mathbf{v}_{n} \cdot \hat{\mathbf{k}} / \hbar k$, along the Fermi contour of the first subband, for Sample 2 in the presence of a $10 T$ parallel magnetic field (solid line). Also included for comparison is the function $m^{*} v_{n} / \hbar k$ (dashed line).

FIG. 4. The centroid of the first subband wave function relative to the interface, as a function of angular position on the Fermi contour. The results are for Sample 2 in the presence of a $10 T$ parallel magnetic field.

FIG. 5. The angular scattering rate $\bar{P}_{11}(\phi, \Delta \phi)$ of the first subband, for Sample 2 with a $10 T$ parallel magnetic field. The scattering rate is normalized by the small-angle scattering rate at $\phi=\pi$. The curves are labelled by the scattering angle $\Delta \phi$, with the solid curves corresponding to positive scattering angles and the dashed curves to negative angles.

FIG. 6. The first subband quantum lifetime, as a function of the angular position on the Fermi contour, for Sample 1 in the presence of a $10 T$ parallel magnetic field. The solid line is the full calculation using the $k_{y}$-dependent wavefunctions, while the dashed line has been computed using only the constant zero field wavefunctions as described in the text.

FIG. 7. As in Figure 6, but for a range of parallel magnetic field strengths. 
FIG. 8. The multiple-subband quantum lifetimes $\tau_{n n^{\prime}}(\phi)$ for Sample 2 in the presence of a 5 T parallel magnetic field, as a function of the angular coordinate.

FIG. 9. The effective transport lifetime of Sample 1 as a function of the angular coordinate, for an applied electric field parallel to the in-plane magnetic field. The lifetime has been normalized by the zero-field transport lifetime of the first subband. The curves are labelled by the parallel magnetic field.

FIG. 10. Variation of the magnetoresistance as a function of the in-plane magnetic field. The labels $x x$ and $y y$ correspond to $\mathbf{J} \| \mathbf{B}_{\|}$and $\mathbf{J} \perp \mathbf{B}_{\|}$, respectively.

FIG. 11. The transverse magnetoresistance for a series of parallel magnetic field strengths. The parameters characterizing this sample are described in the text. The second subband depopulates at a parallel magnetic field of approximately $4 T$. The curves have been offset arbitrarily for clarity, but the scale of resistance variation is the same for each.

FIG. 12. The transverse magnetoresistance for $\mathbf{J} \| \mathbf{B}_{\|}(x x)$ and $\mathbf{J} \perp \mathbf{B}_{\|}$(yy). (a) and (b) correspond to Samples 1 and 2, respectively, both at a field $B_{\|}=5 T$.

FIG. 13. The normalized transverse magnetoresistance as a function of parallel magnetic field strength. The different curves are labelled by the acceptor density $n_{A}$, in units of $10^{11} \mathrm{~cm}^{-2}$. All samples have the same electron density of $7.1 \times 10^{11} \mathrm{~cm}^{-2}$. The low-field termination of the curves occurs at the point where the second subband becomes populated.

FIG. 14. (a) The normalized magnetoresistance as a function of $n_{A}$ at three different parallel magnetic fields, for the same set of samples as in Figure 11. (b) As in (a), but for parallel magnetic field of $10 T$ and varying electron gas density, $n_{T}$, in units of $10^{11} \mathrm{~cm}^{-2}$. 

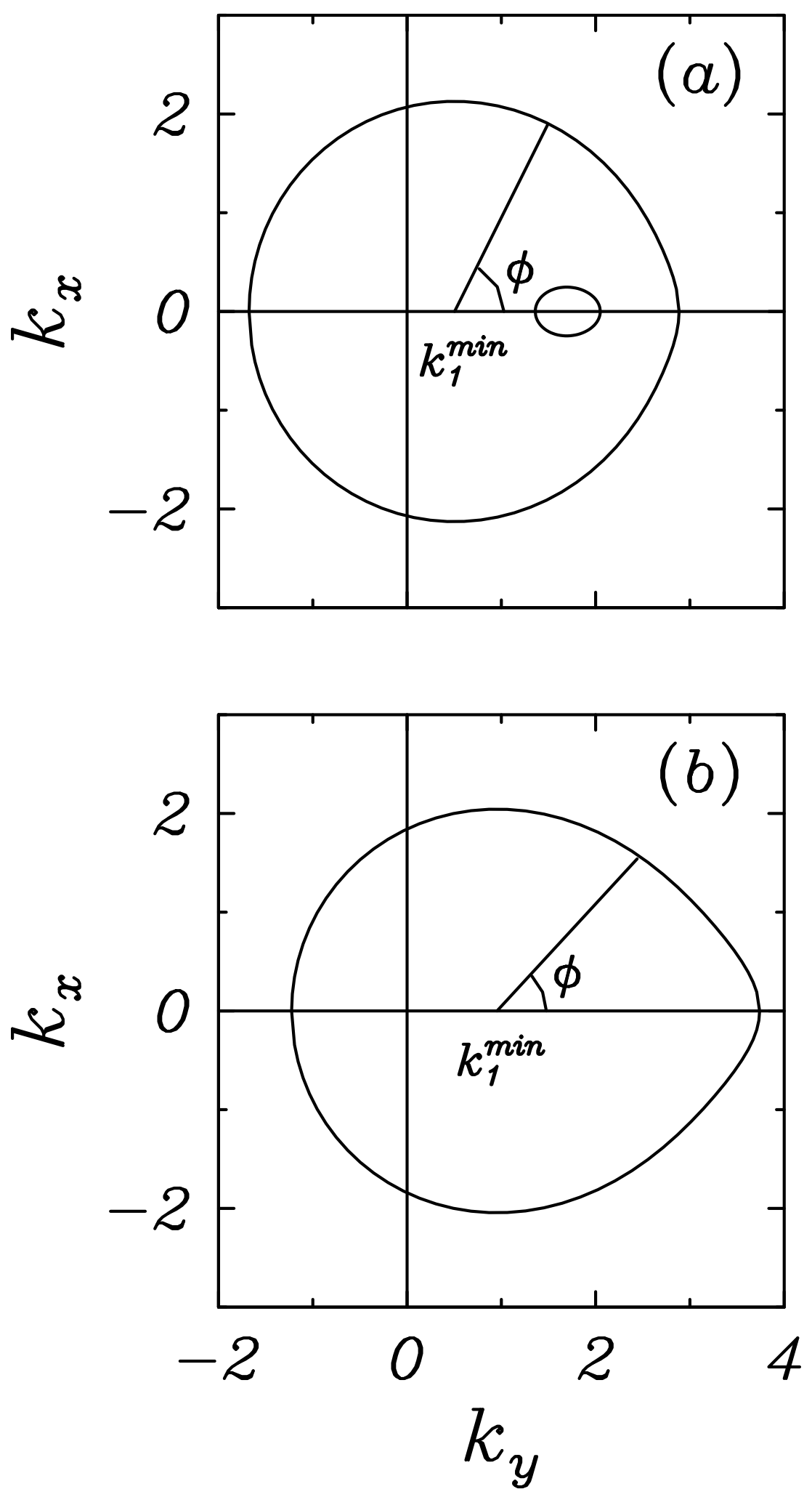


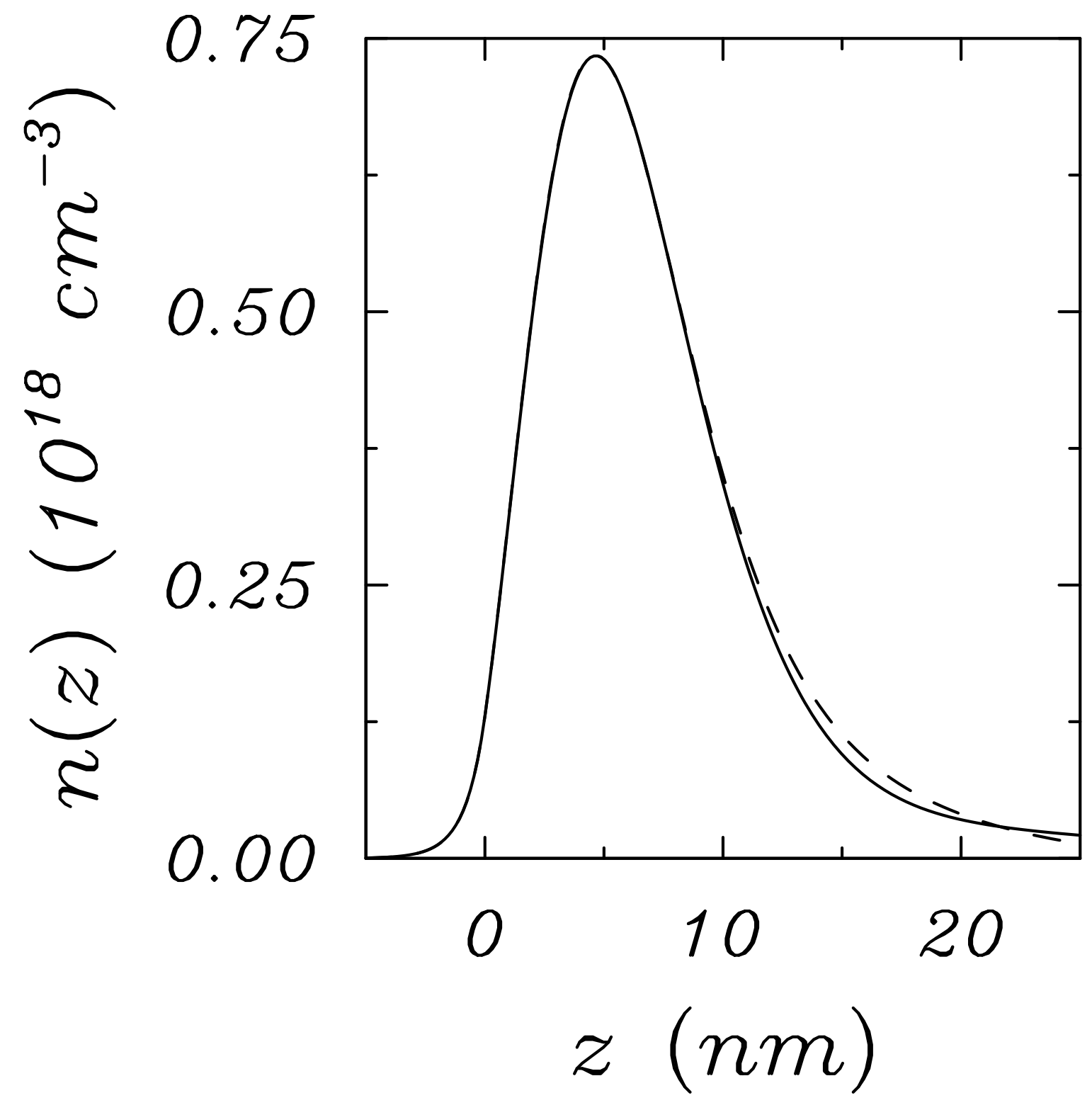




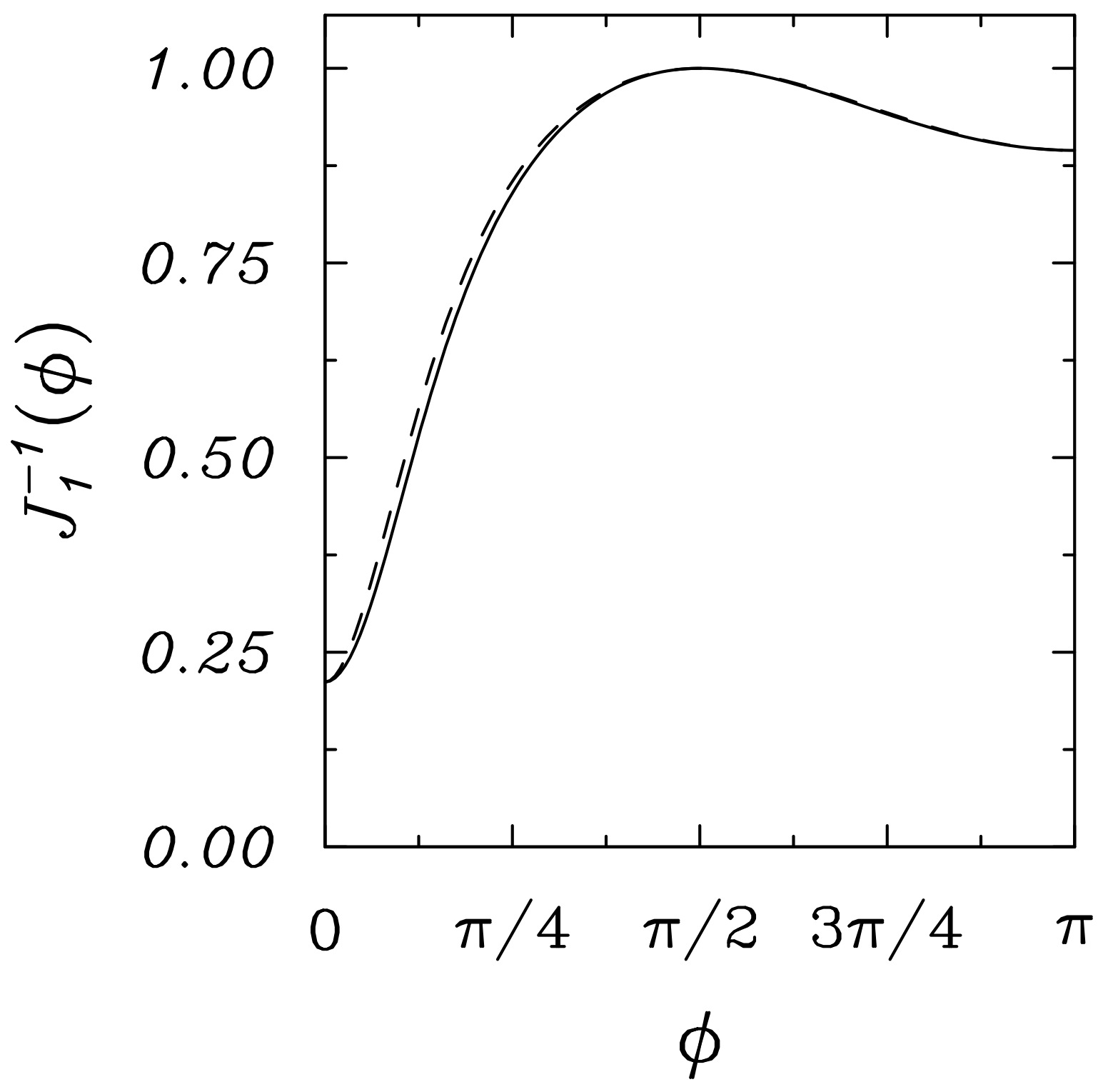




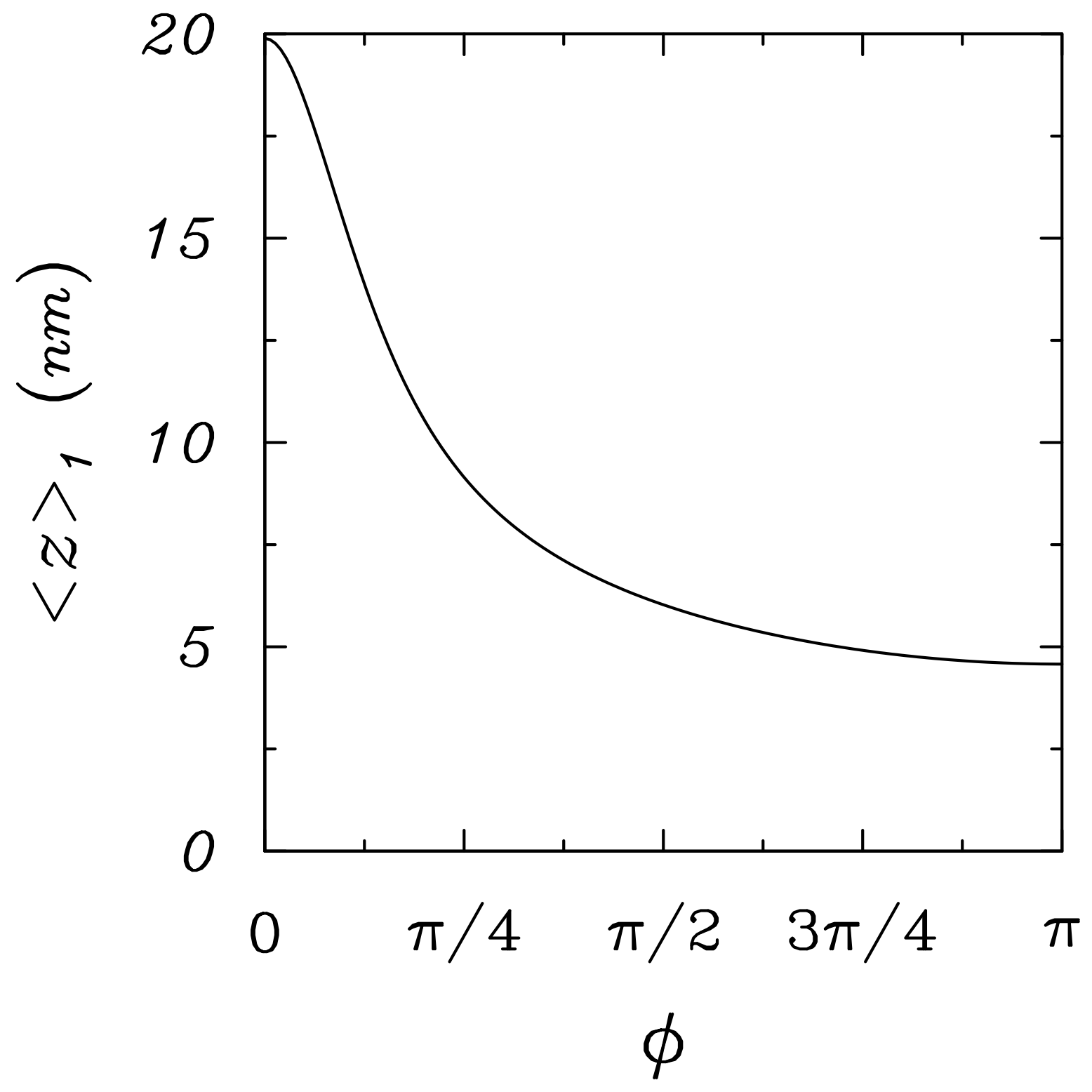




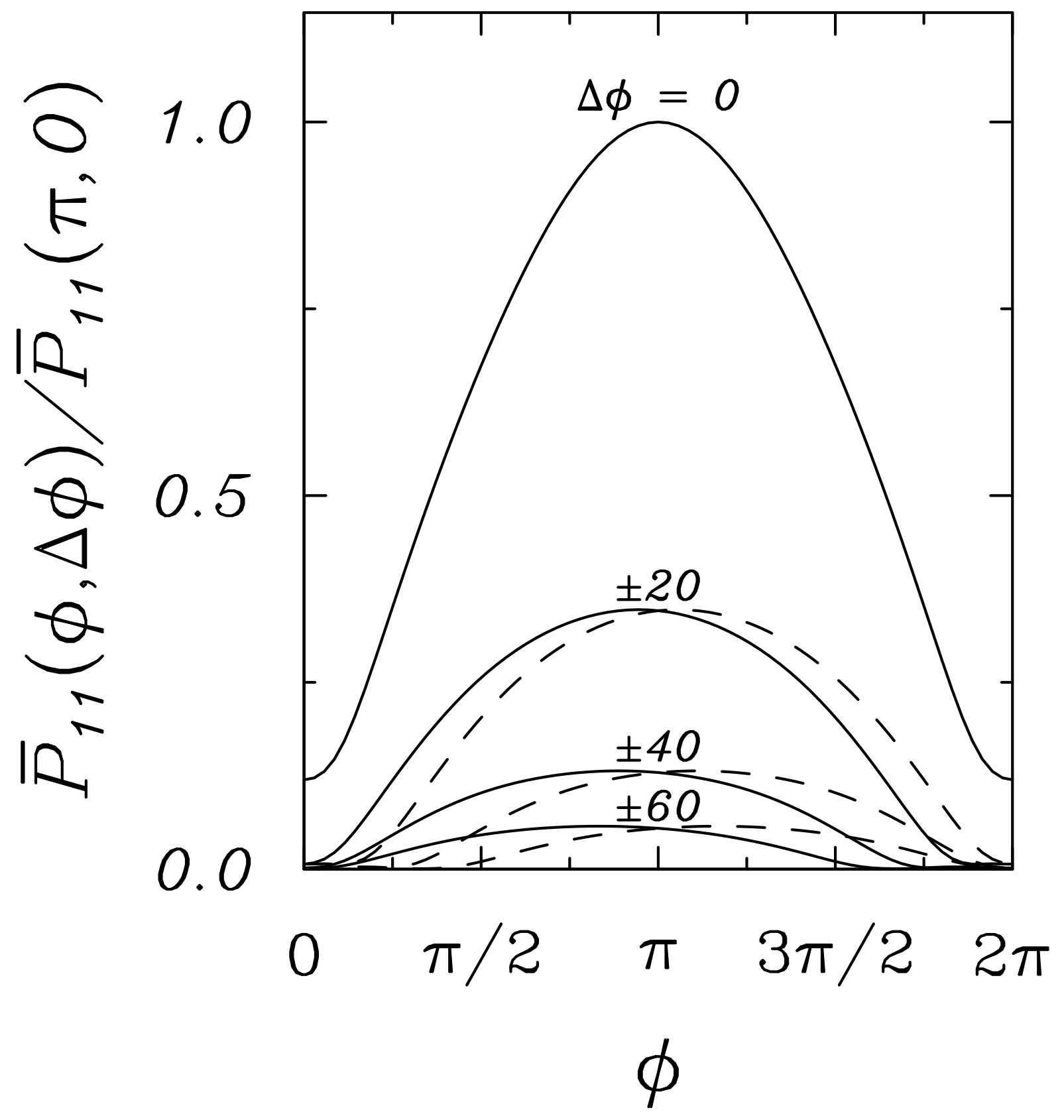




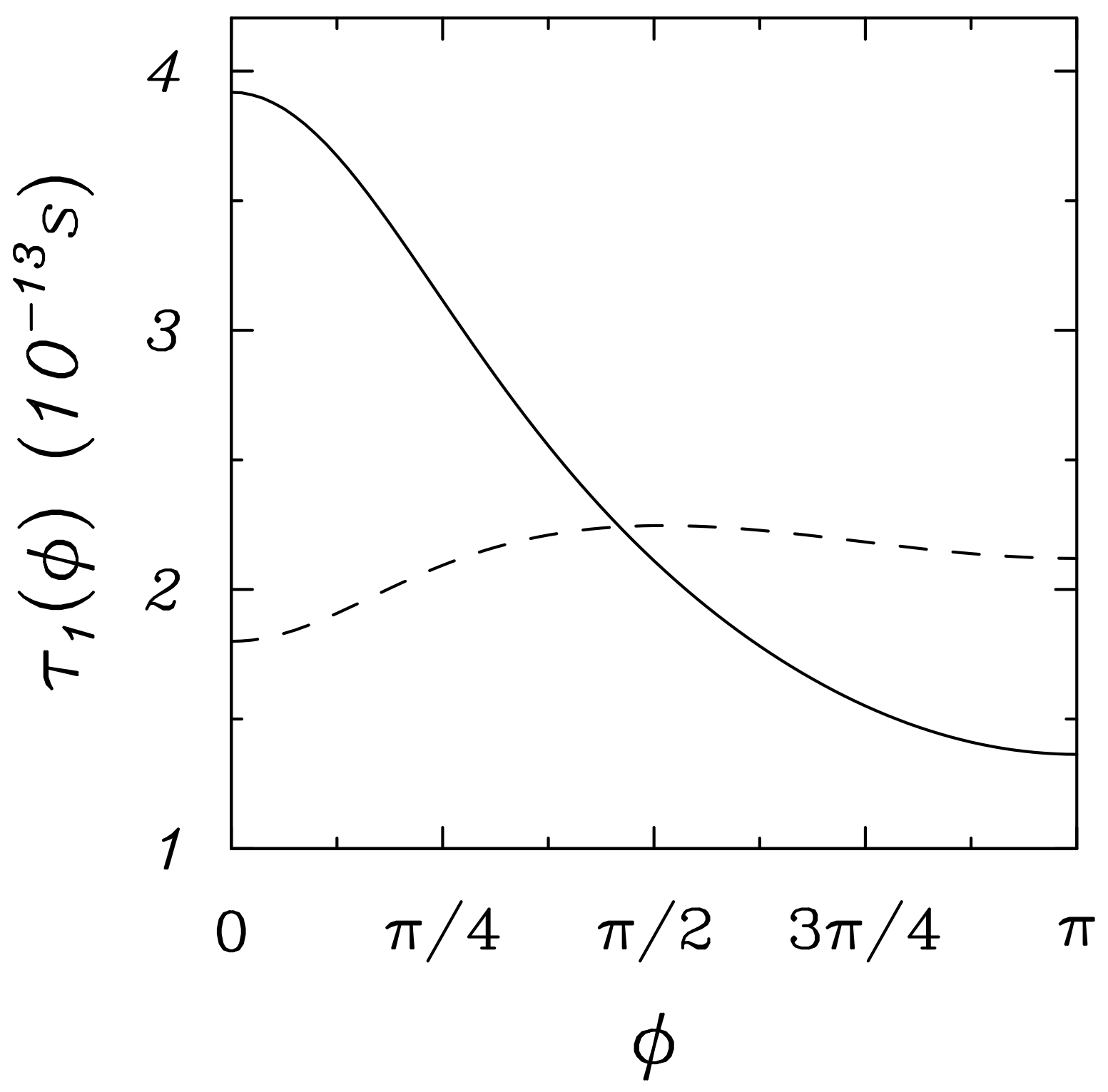




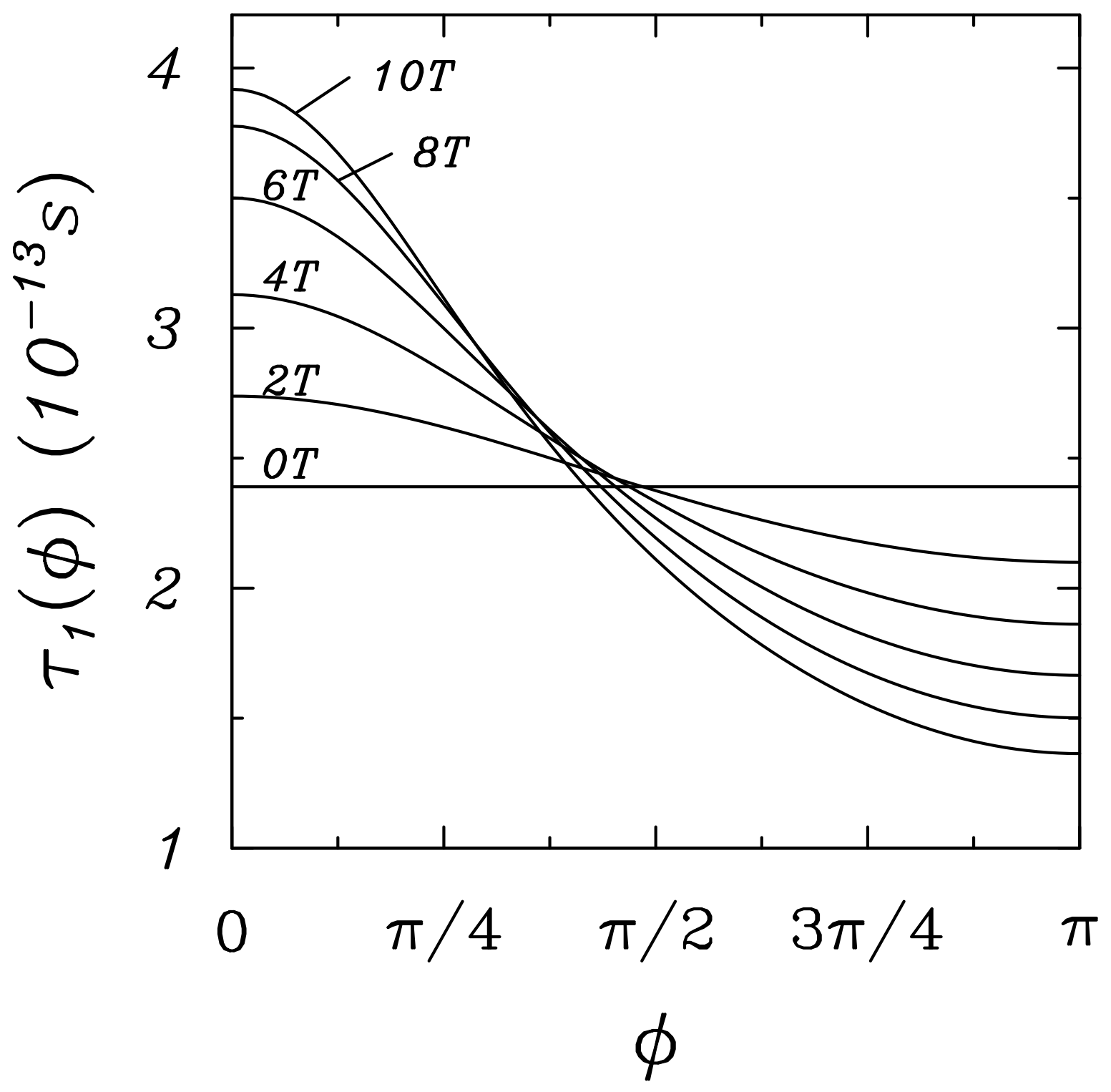




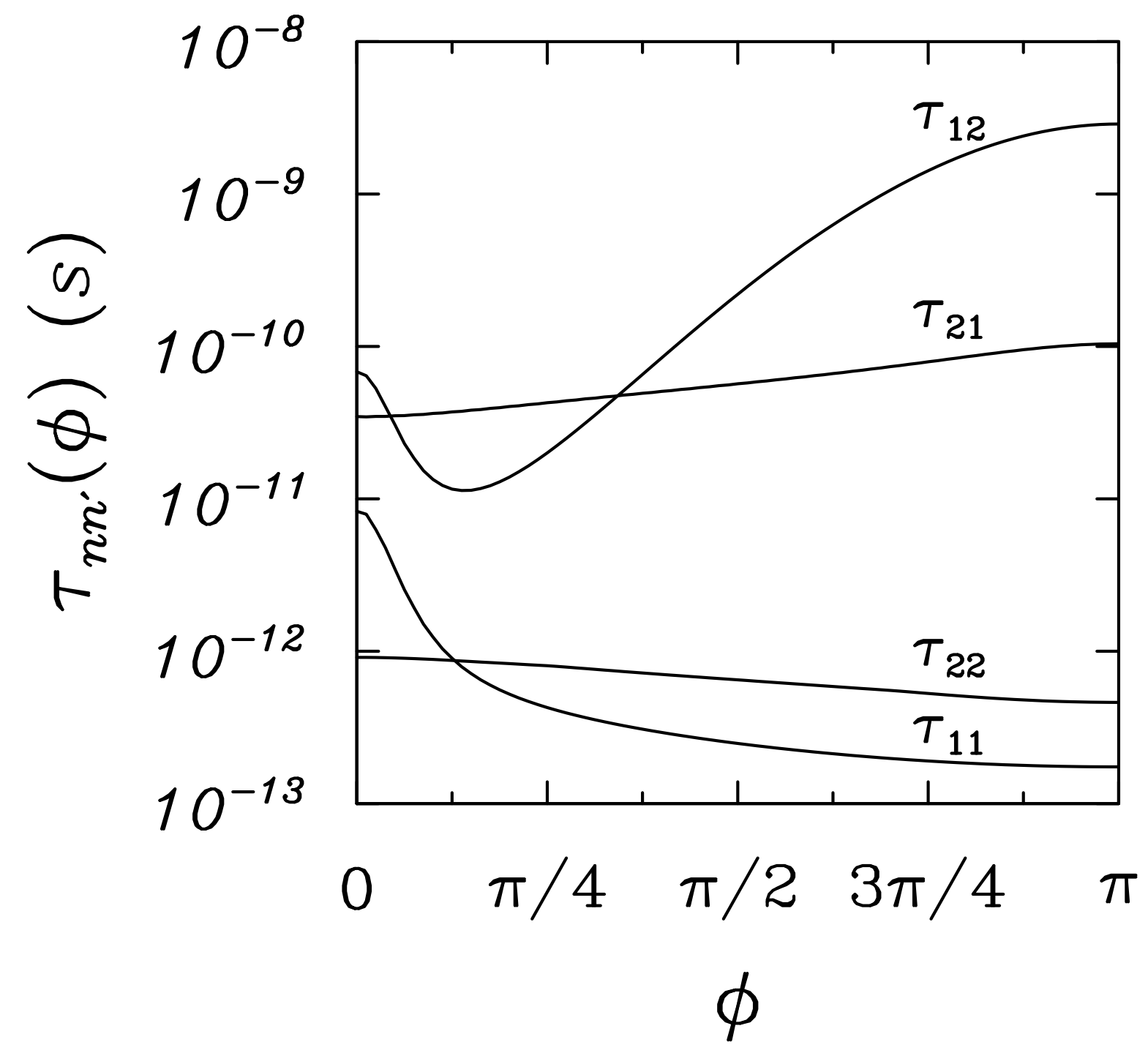




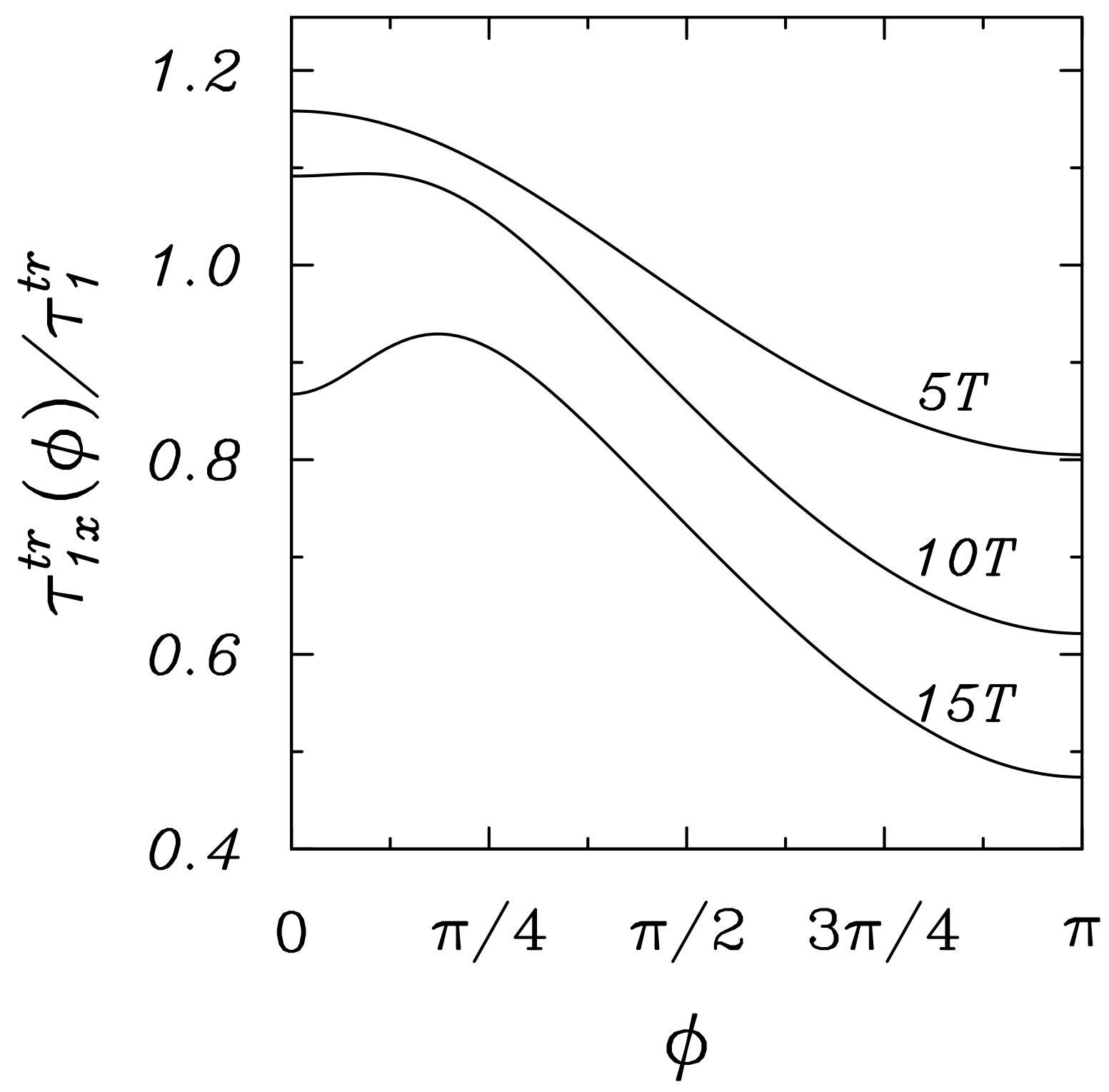




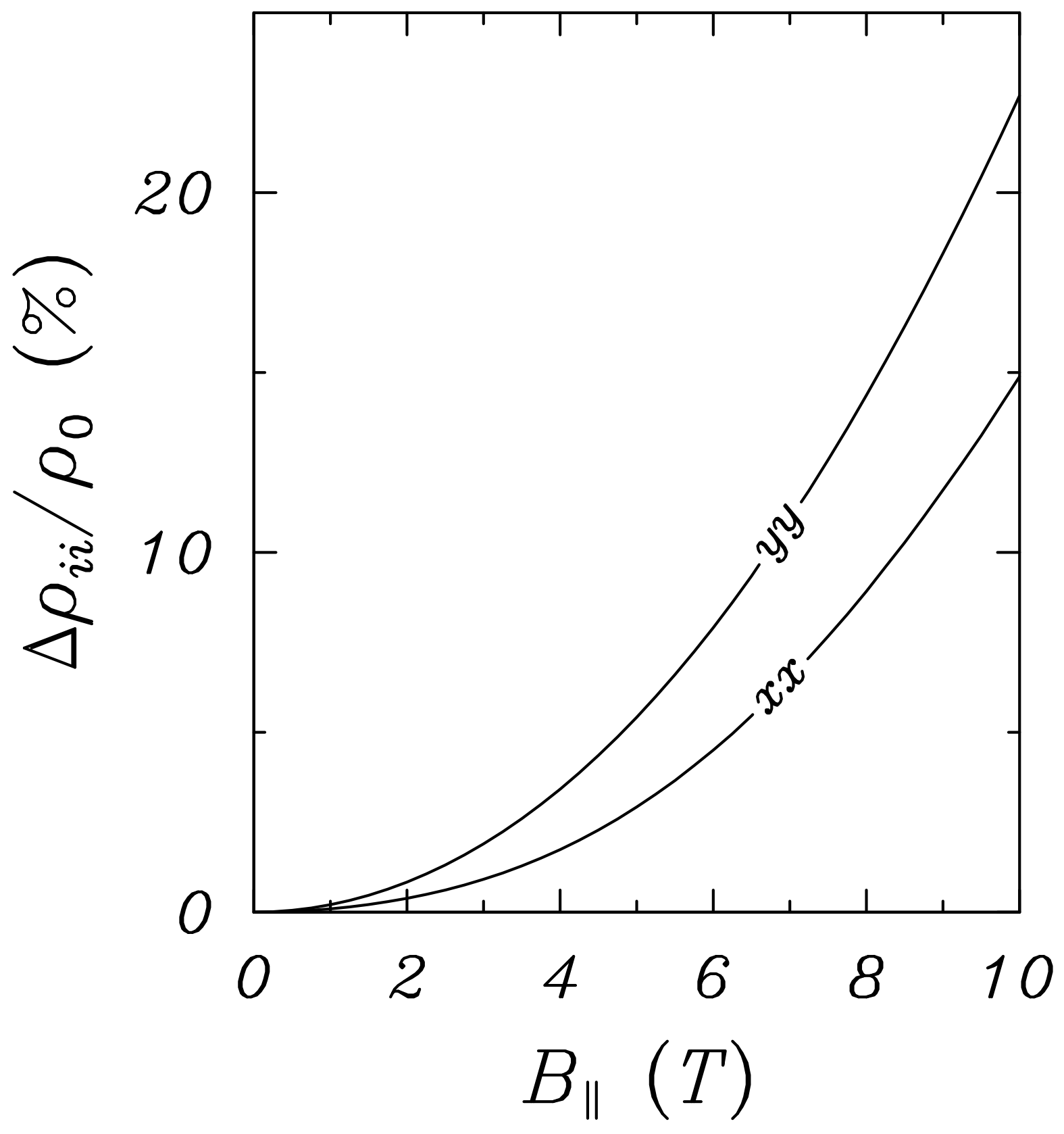




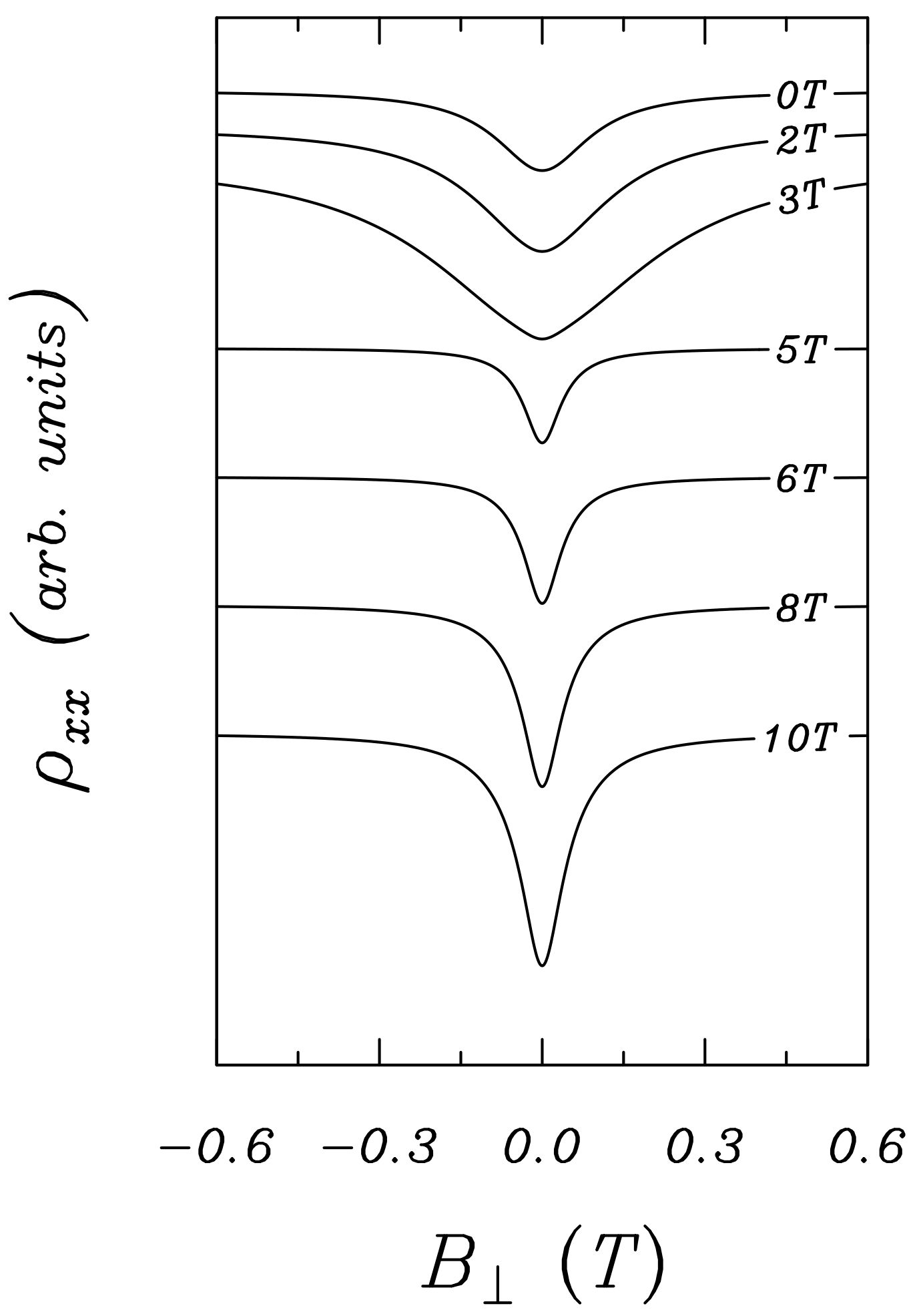




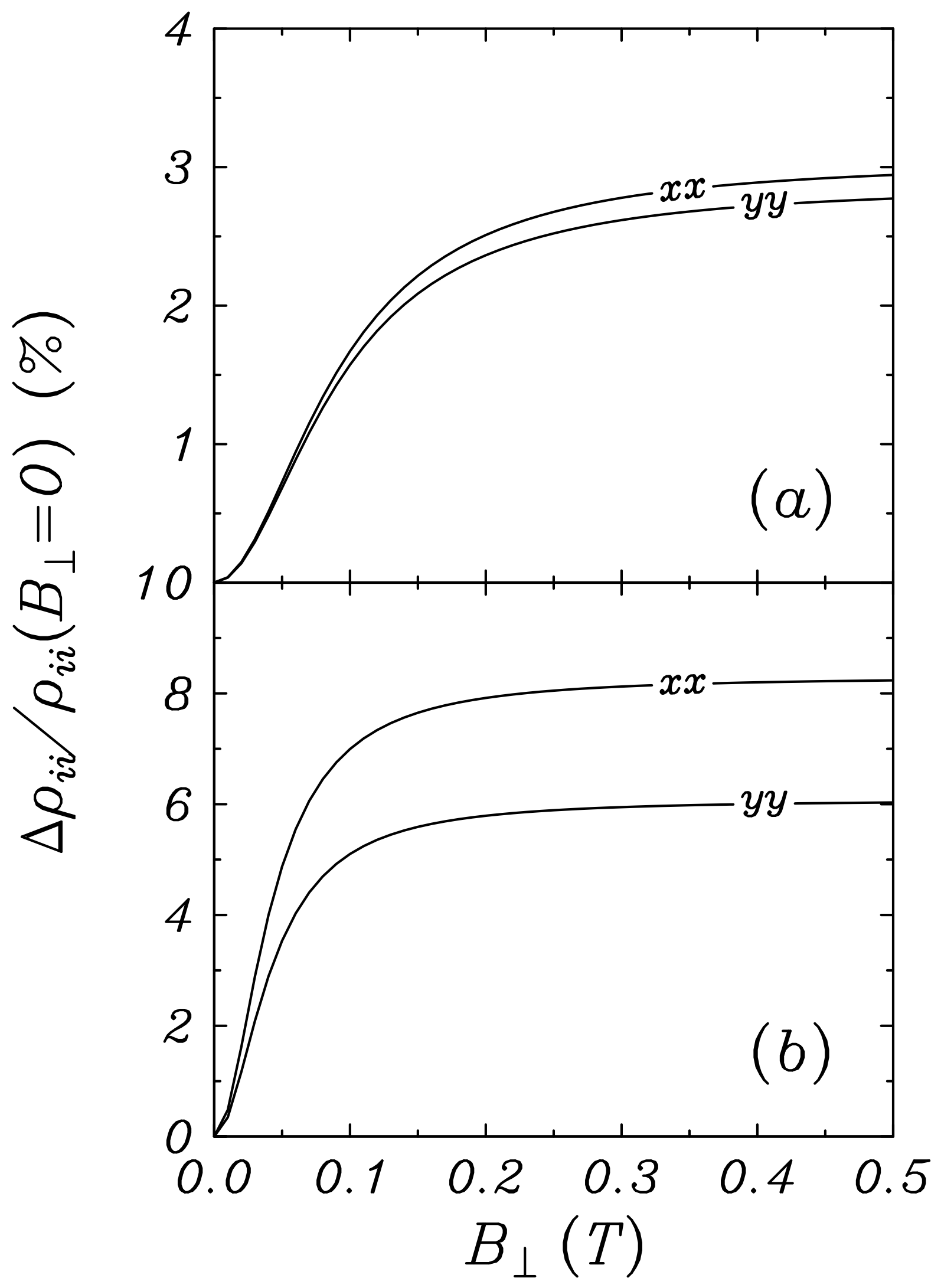




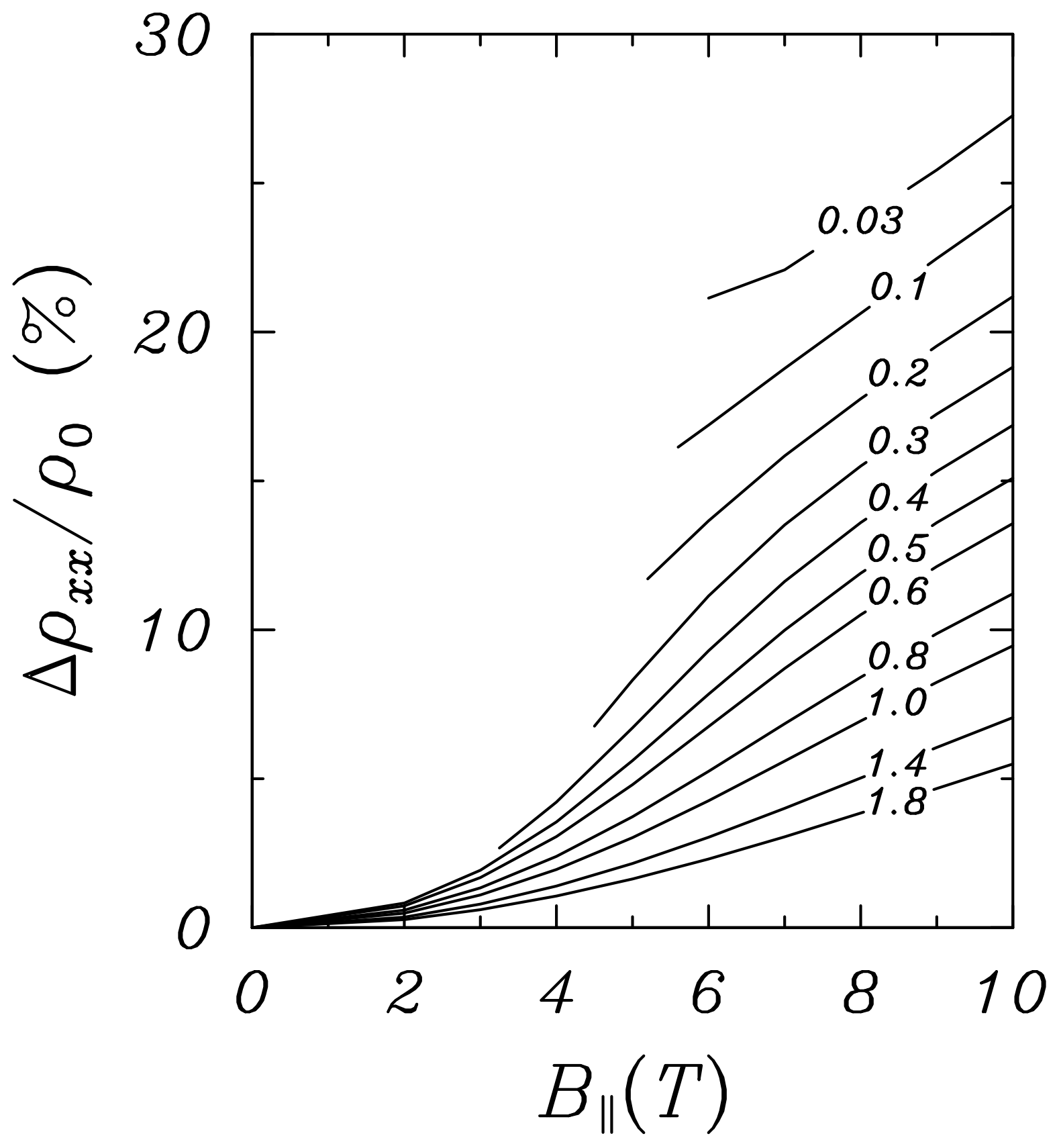




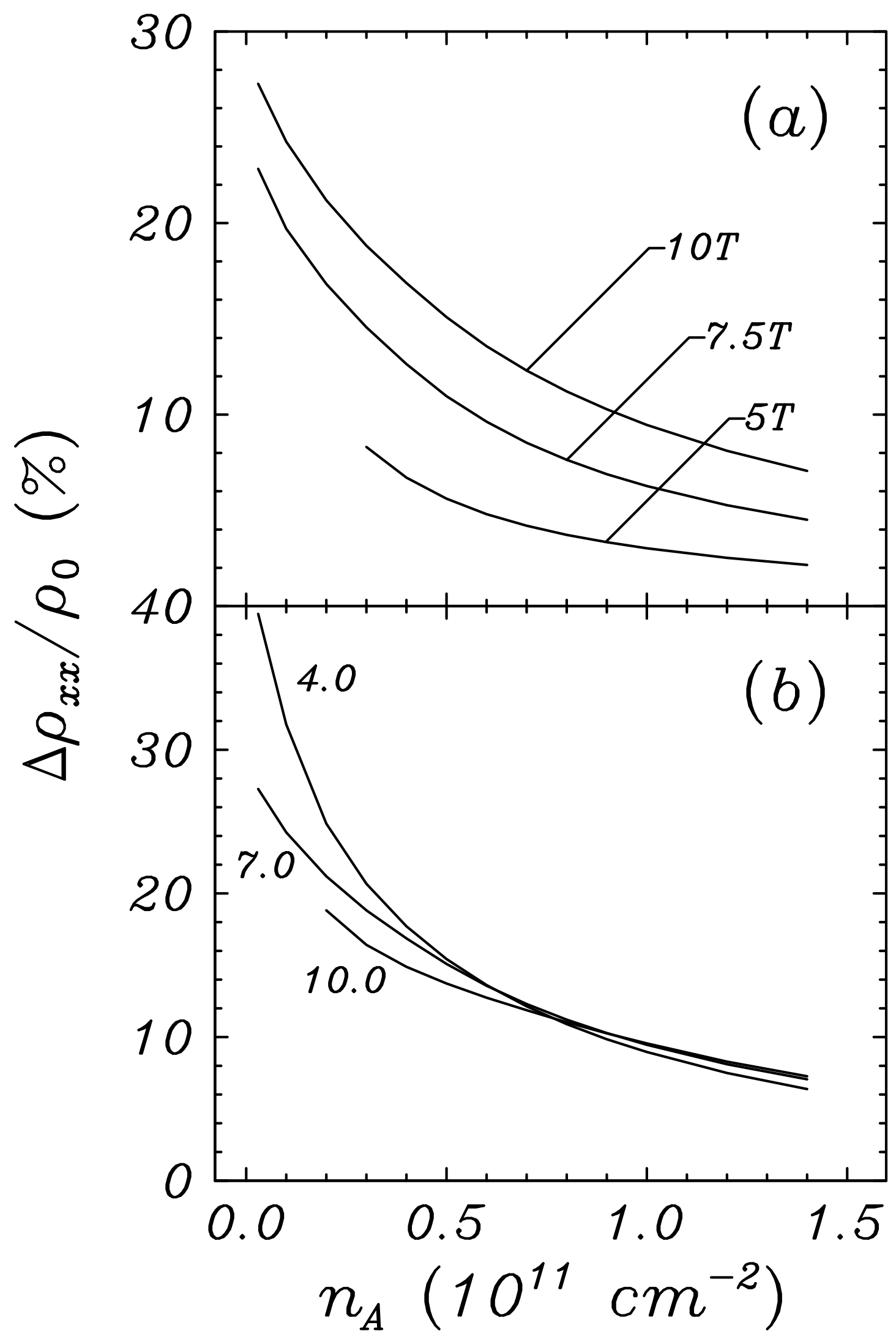

